\title{
Multichannel Single-Sideband SCM/DWDM Transmission Systems
}

\author{
W. H. Chen and Winston I. Way, Fellow, IEEE
}

\begin{abstract}
To understand the transmission limitation of multiple narrow single-sideband subcarrier-multiplexed (SSB/SCM) signals, this paper first presents a closed-form analysis to predict Mach-Zehnder intensity modulator-induced composite triple beat (CTB), and linear-fiber-dispersion-induced composite second-order (CSO) and CTB distortions. To combine SSB/SCM with dense-wavelength-division-multiplexing (DWDM) systems, analytical and numerical tools, which are not constrained by any wavelength spacing and modulation frequencies, are used to analyze cross-phase modulation-induced crosstalk. All the analytical and numerical results are verified by computer simulations. Several multichannel SSB/SCM/DWDM systems with transport capacities of 10 or $20 \mathrm{~Gb} / \mathrm{s}$ per wavelength, with a wavelength spacing of 25, 50, and $100 \mathrm{GHz}$, are also studied in this paper to understand the fundamental transmission limitations.
\end{abstract}

Index Terms-Cross-phase modulation (XPM), dense-wavelength-division multiplexing (DWDM), fiber dispersion, subcarrier multiplexing (SCM).

\section{INTRODUCTION}

$\mathbf{I}$ $\mathrm{N}$ a conventional subcarrier-multiplexed (SCM) transmission system, lower and upper subcarrier sidebands appear on both sides of an optical carrier, and their phases tend to rotate with respect to each other due to the chromatic dispersion in a standard single-mode fiber (SMF). At a certain transmission distance, the two sidebands could be $180^{\circ}$ out of phase, and after down-conversion through a square-law photodetector, the signal is canceled out. This kind of "fading" phenomenon could periodically occur at different transmission distances along a fiber link. Single-sideband SCM (SSB/SCM) modulation eliminates the energy on one side of the optical carrier, and consequently its chromatic dispersion tolerance is significantly enhanced-limited only by the symbol rate of the individual subcarriers. As a result, SSB/SCM has been used in wireless-optical fiber links operating at millimeter-wave subcarriers to carry a narrow-band signal, such as $51.8 \mathrm{Mb} / \mathrm{s}$ at $12 \mathrm{GHz}$ over $80 \mathrm{~km}$ of SMF [1], and $155 \mathrm{Mb} / \mathrm{s}$ at $38 \mathrm{GHz}$ over $50 \mathrm{~km}$ of SMF [2]. SSB/SCM has also been applied to a transmission system carrying multiple channels (e.g., a system with 70 channels of 6-MHz amplitude modulation-vestigial sideband (AM-VSB) at $28 \mathrm{GHz}$ over $23.8 \mathrm{~km}$ of SMF was studied [3]). However, only simulation results were provided in [3], and no clear analytical guideline was given.

Manuscript received October 2, 2003; revised March 22, 2004.

W. H. Chen is with the Department of Communications Engineering, National Chiao-Tung University, Hsinchu, Taiwan 30010, R.O.C.

W. I. Way is with the OpVista Inc., Irvine, CA 92618 USA.

Digital Object Identifier 10.1109/JLT.2004.829210
This paper first provides a study of the fundamental transmission limitations in a single-wavelength multichannel SSB/SCM system. These include fiber-dispersion-induced second- and third-order nonlinear distortions and the effect of self-phase modulation (SPM). Then, it is investigated whether dense-wavelength-division multiplexing (DWDM) can be used in combination with the multichannel SSB/SCM modulation technique. Computer simulations, numerical calculations, and fundamental analytical tools are provided to understand the effect of cross-phase modulation (XPM) [4] and XPM-to-intensity-modulation (IM) conversion via chromatic dispersion, for various wavelength spacing and modulation frequencies. We also present a few metro optical network case studies to understand the applicability of SSB/SCM/DWDM. The fundamental analysis and numerical calculations used in this paper can be easily extended to multichannel wireless-optical fiber links.

\section{SySTEM CONFIGURATION}

Fig. 1 illustrates the basic SSB/SCM/DWDM system configuration. Multiple SCM channels from digital modems at a single intermediate frequency, with or without foward-error correction (FEC) codecs, are frequency converted and filtered to a series of equally spaced subcarrier frequencies $\left(f_{1}, f_{2}, \ldots f_{k}\right)$. The subcarrier channels are then combined together, creating a single composite radio-frequency (RF) signal consisting of $k$ independent subcarrier channels. Subsequently, the composite RF signal is used to modulate an ITU wavelength via a dual-drive Mach-Zehnder interferometer (MZI) electrooptical modulator [1].

The composite modulating signal from an SCM block (see Fig. 1) was applied to both electrodes of the MZI modulator, with a $\pi / 2$ relative phase shift between the arms. A dc bias sets the modulator at a quadrature point. Note that if a $\pi / 2$ phase shift cannot be met over a wide frequency range, multiple phase shifters will have to be built. Thereafter, a number of wavelengths $\left(\lambda_{1}, \lambda_{2}, \ldots \lambda_{N}\right)$, each carrying plural single-sided SCM signals, are combined by a conventional DWDM multiplexer onto the same fiber. At the receiving terminal, these wavelengths are demultiplexed and a high-speed photodetector converts the composite SCM signals in a single wavelength back to their original RF frequencies. The composite SCM signal is then separated into constituent signals by a sequence of electrical frequency converters, filters, demodulators, and decoders. 


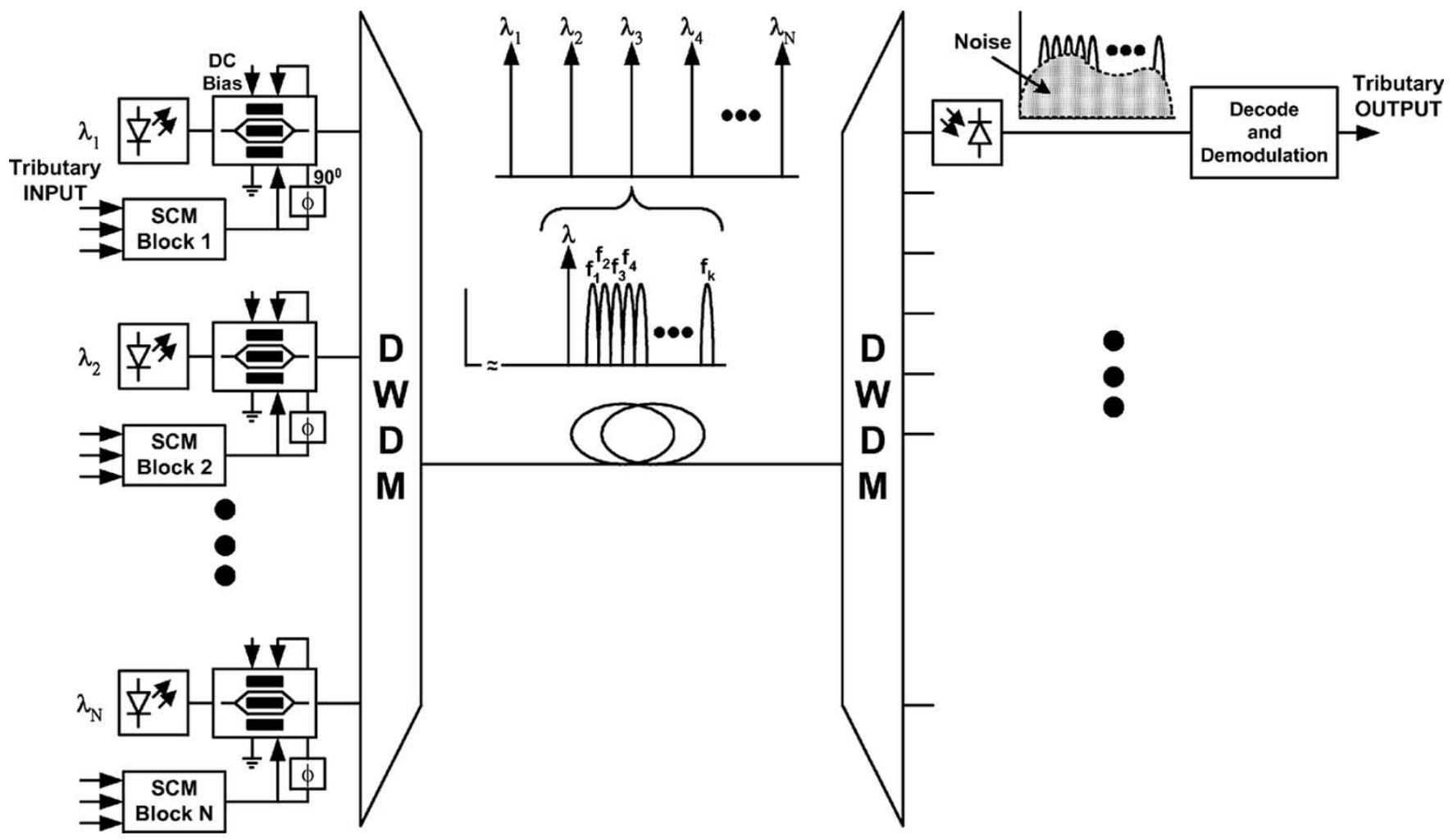

Fig. 1. SSB/SCM/DWDM system.

\section{NONLINEAR DisTORTIONS IN AN SSB/SCM TRANSMISSION SYSTEM-SINGLE SUBCARRIER CHANNEL}

\section{A. Third-Order Harmonic Due to MZI Modulator}

The output electrical field of a differential dual-electrode MZI modulator can be written as

$$
\begin{gathered}
E_{\text {out }}=\frac{E_{\text {in }}}{\sqrt{2}}\left[\cos \left(\omega_{o} t+\phi_{1}\right)+\cos \left(\omega_{o} t-\phi_{2}\right)\right] \\
=\frac{E_{\text {in }}}{\sqrt{2}}\left[\left(\cos \phi_{1}+\cos \phi_{2}\right) \cdot \cos \omega_{o} t\right. \\
\left.-\left(\sin \phi_{1}-\sin \phi_{2}\right) \cdot \sin \omega_{o} t\right]
\end{gathered}
$$

where $\omega_{0}$ is the lightwave carrier angular frequency; $E_{\text {in }}$ and $E_{\text {out }}$ are the input and output electrical fields of the MZI modulator, respectively; and $\phi_{1}$ and $\phi_{2}$ are the voltage-induced phase shifts of the two arms of the MZI modulator, both of which can be represented in the form of $\left(\pi \cdot V_{\mathrm{dc}} / V_{\pi, \mathrm{dc}}\right)+\left(\pi \cdot V_{\mathrm{ac}}(t) / V_{\pi, \mathrm{ac}}\right)$ (where $V_{\pi, \mathrm{dc}}$ and $V_{\pi, \mathrm{ac}}$ are the voltages required at the dc-bias port and RF input ports, respectively, to cause a $180^{\circ}$ phase shift between the two interferometric arms). $V_{\mathrm{dc}}$ and $V_{\mathrm{ac}}(t)$ are the dc-bias voltage and RF drive signal, respectively. The opposite signs of $\phi_{1}$ and $\phi_{2}$ in the two cosine terms of (1) are attributed to the "differential" electrode configuration of the MZI modulator.

For SSB/SCM modulation, the RF signals at the two electrodes of a modulator must be a Hilbert transform pair, which means that one of the two RF signals has to pass through a $90^{\circ}$ phase shifter. Furthermore, the modulator is dc biased at a quadrature point of the nonlinear electrical-to-optical (E/O) transfer curve of the modulator to avoid second-order nonlinear distortions. Therefore, if the modulator is modulated by $\cos (\Omega t)$, where $\Omega$ is a modulating RF frequency, $\phi_{1}$ and $\phi_{2}$ can be represented as $\pi / 2+m \cdot \cos (\Omega t)$ and $m \cdot \sin (\Omega t)$, respectively, where $m=\pi \cdot V_{\mathrm{ac}} / V_{\pi \text {,ac }}$ is the root-mean-square optical modulation index (rms OMI). Note that $m^{2} / 2$ is the normalized electrical power of the subcarrier channel at an RF input port of the MZI modulator. The output electrical field of the single RF-modulated optical signal can then be obtained by rewriting (1) as (3), shown at the bottom of the next page, where we have used Bessel function expansion to obtain (3), and $J_{k}(\cdot)(k=1,2,3 \ldots)$ is the Bessel function of the first kind $k$ th order. As expected, the electrical field is composed of, in addition to the single-sided fundamental modulating frequency, an infinite number of harmonics of the modulating frequency. The even harmonics are double-sided, whereas the odd harmonics consist of alternative upper or lower sidebands at different orders. Fig. 2 shows the optical spectrum of a single channel SSB/SCM signal.

If only transmission loss is considered, the detected photocurrent is given by

$$
\begin{aligned}
I=\Re & \cdot \eta \cdot\left|E_{\text {out }}\right|^{2} \approx \frac{\Re \eta\left|E_{\text {in }}\right|^{2}}{2} \\
& \cdot\left\{J_{0}^{2}(m)-2 \sqrt{2} J_{0}(m) J_{1}(m) \cdot \cos \left(\Omega t-\frac{\pi}{4}\right)\right. \\
& +2 \sqrt{2}\left[J_{0}(m) J_{3}(m)-J_{1}(m) J_{2}(m)\right] \\
& \left.\cdot \cos \left(3 \Omega t+\frac{\pi}{4}\right)+\cdots\right\}
\end{aligned}
$$

where $\Re$ is the photodiode responsivity, and $\eta$ is the optical power attenuation. The first term $\Re \eta\left|E_{\text {in }}\right|^{2} J_{0}^{2}(m) / 2=P_{r} \Re$ is the average photocurrent, and $P_{r}$ is the received optical power. Note that the result contains no even-order harmonics, as can 


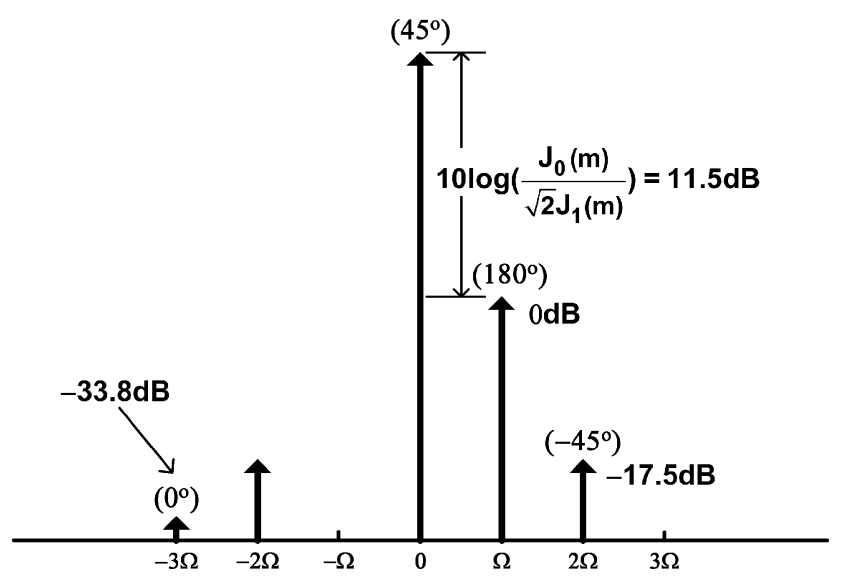

Fig. 2. Optical spectrum of a single-channel SSB/SCM signal with $m=0.1$ (note that $\omega_{0}$ is subtracted from all frequency components).

be seen in (4). The third-order harmonic distortion (HD3) due to the intrinsic nonlinear E/O "cosine" transfer curve of an MZI modulator, while using SSB/SCM modulation without considering fiber dispersions, is given by

$$
\mathrm{HD}_{\mathrm{SSB} / \mathrm{SCM}, \mathrm{MZI}}=\left[\frac{J_{0}(m) J_{3}(m)-J_{1}(m) J_{2}(m)}{J_{0}(m) J_{1}(m)}\right]^{2} .
$$

\section{B. Second- and Third-Order Harmonic Distortions Due to Linear Fiber Dispersion}

Equation (3) can be modified to include the effect of linear fiber dispersion in a transmission link. The electrical field of an SSB/SCM signal after propagating through a SMF with a length $z$ is given by (6), shown at the bottom of the page, where $\beta_{\omega}$ is the propagation constant at a frequency $\omega$, which can be expanded around an optical carrier frequency $\omega_{o}$ as

$$
\beta_{\omega}=\beta_{0}+\beta_{1}\left(\omega-\omega_{o}\right)+\frac{1}{2} \beta_{2}\left(\omega-\omega_{o}\right)^{2}
$$

where the higher order dispersion terms are neglected. $\beta_{1}$ and $\beta_{2}$ denote the first and second derivatives of the propagation constant with respect to the optical carrier frequency, respectively. The propagation phase shift $\beta_{\omega} z$ for each optical subcarrier term is different due to the change in the refractive index as a function of the frequency. The SSB/SCM signal propagates in a fiber link with a group velocity $1 / \beta_{1}$, and the fiber dispersion $D$ is related to the chromatic dispersion parameter $\beta_{2}$ by $D=-2 \pi c \beta_{2} / \lambda^{2}$, where $\lambda$ is the operating wavelength and $c$ the velocity of light in vacuum.

According to (6), the detected photocurrent can be written as (8), shown at the bottom of the next page, where

$C_{1}=$

$J_{0}(m) J_{3}(m) \cos \left(\frac{1}{2} \beta_{2}(3 \Omega)^{2} z\right)-J_{1}(m) J_{2}(m) \cos \left(\frac{1}{2} \beta_{2} 3 \Omega^{2} z\right)$.

and

$C_{2}=$

$$
J_{0}(m) J_{3}(m) \sin \left(\frac{1}{2} \beta_{2}(3 \Omega)^{2} z\right)-J_{1}(m) J_{2}(m) \sin \left(\frac{1}{2} \beta_{2} 3 \Omega^{2} z\right)
$$

Note that higher order harmonics have been neglected in (8). Based on (8), we can see that when SSB/SCM modulation is

$$
\begin{aligned}
E_{\mathrm{out}}=\frac{E_{\mathrm{in}}}{\sqrt{2}}\{ & {\left[\cos \left(\frac{\pi}{2}+m \cos \Omega t\right)+\cos (m \sin \Omega t)\right] \cdot \cos \omega_{o} t } \\
& \left.-\left(\sin \left(\frac{\pi}{2}+m \cos \Omega t\right)-\sin (m \sin \Omega t)\right) \cdot \sin \omega_{o} t\right\} \\
=\frac{E_{\text {in }}}{\sqrt{2}}\{ & \sqrt{2} J_{0}(m) \cdot \cos \left(\omega_{o} t+\frac{\pi}{4}\right)-2 J_{1}(m) \cdot \cos \left[\left(\omega_{o}+\Omega\right) \cdot t\right] \\
& +\sqrt{2} J_{2}(m) \cdot\left\{\cos \left[\left(\omega_{o}+2 \Omega\right) \cdot t-\frac{\pi}{4}\right]+\cos \left[\left(\omega_{o}-2 \Omega\right) \cdot t-\frac{\pi}{4}\right]\right\} \\
& +2 J_{3}(m) \cdot \cos \left[\left(\omega_{o}-3 \Omega\right) \cdot t\right] \\
& +\sqrt{2} J_{4}(m) \cdot\left\{\cos \left[\left(\omega_{o}+4 \Omega\right) \cdot t+\frac{\pi}{4}\right]+\cos \left[\left(\omega_{o}-4 \Omega\right) \cdot t+\frac{\pi}{4}\right]\right\} \\
& \left.-2 J_{5}(m) \cdot \cos \left[\left(\omega_{o}+5 \Omega\right) \cdot t\right]+\cdots\right\}
\end{aligned}
$$

$$
\begin{aligned}
E_{\text {out }, D}= & \frac{E_{\text {in }}}{\sqrt{2}}\left\{\sqrt{2} J_{0}(m) \cdot \cos \left(\omega_{o} t+\frac{\pi}{4}-\beta_{\omega_{o}} z\right)-2 J_{1}(m) \cdot \cos \left[\left(\omega_{o}+\Omega\right) \cdot t-\beta_{\omega_{o}+\Omega} z\right]\right. \\
& +\sqrt{2} J_{2}(m) \cdot\left\{\cos \left[\left(\omega_{o}+2 \Omega\right) \cdot t-\frac{\pi}{4}-\beta_{\omega_{o}+2 \Omega} z\right]+\cos \left[\left(\omega_{o}-2 \Omega\right) \cdot t-\frac{\pi}{4}-\beta_{\omega_{o}-2 \Omega} z\right]\right\} \\
& +2 J_{3}(m) \cdot \cos \left[\left(\omega_{o}-3 \Omega\right) \cdot t-\beta_{\omega_{o}-3 \Omega} z\right] \\
& +\sqrt{2} J_{4}(m) \cdot\left\{\cos \left[\left(\omega_{o}+4 \Omega\right) \cdot t+\frac{\pi}{4}-\beta_{\omega_{o}+4 \Omega} z\right]+\cos \left[\left(\omega_{o}-4 \Omega\right) \cdot t+\frac{\pi}{4}-\beta_{\omega_{o}-4 \Omega} z\right]\right\} \\
& \left.-2 J_{5}(m) \cdot \cos \left[\left(\omega_{o}+5 \Omega\right) \cdot t-\beta_{\omega_{o}+5 \Omega} z\right]+\cdots\right\}
\end{aligned}
$$




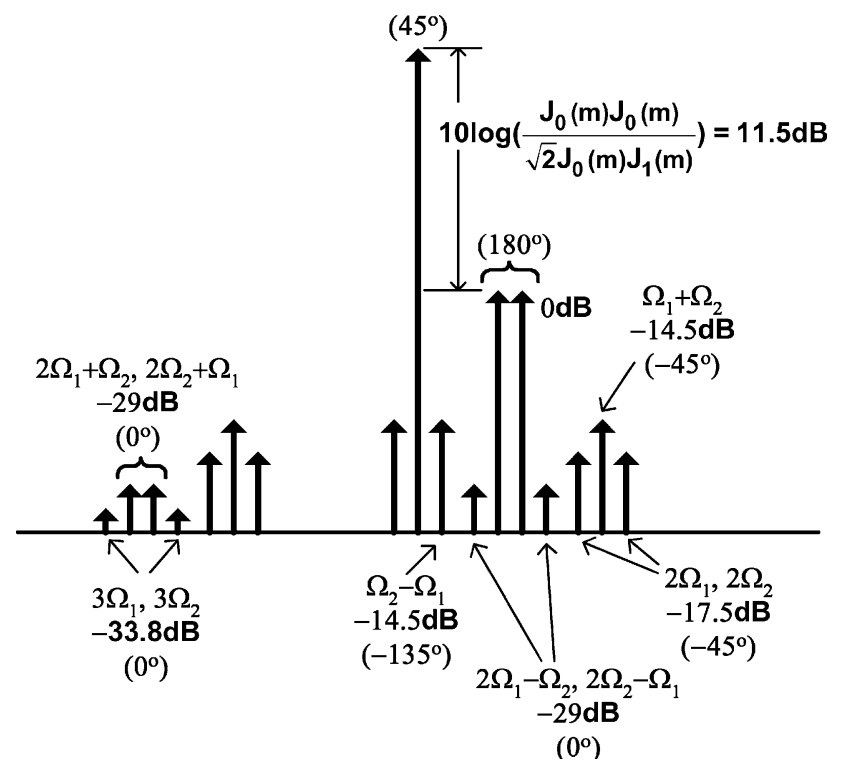

Fig. 3. Optical spectrum of a 2-ch SSB/SCM signal with $m=0.1$ (note that $\omega_{0}$ is subtracted from all frequency components).

used, and a fiber transmission link with a distance $z$ is considered, the normalized second- and third-order harmonic distortions are given by

$$
\begin{aligned}
\mathrm{HD} 2_{\mathrm{SSB} / \mathrm{SCM}, D} & =2\left[\frac{J_{2}(m) \sin \left(\frac{1}{2} \beta_{2}(2 \Omega)^{2} z\right)}{J_{1}(m)}\right]^{2} \\
\mathrm{HD} 3_{\mathrm{SSB} / \mathrm{SCM}, D} & =\frac{C_{1}^{2}+C_{2}^{2}}{J_{0}^{2}(m) J_{1}^{2}(m)} .
\end{aligned}
$$

\section{NONLINEAR DISTORTIONS IN AN SSB/SCM TRANSMISSION SYSTEM-MULTIPLE SUBCARRIER CHANNELS}

\section{A. CTB Due to MZI Modulator}

The derivations in Section III-A can be extended to the case of multiple subcarrier channels, i.e., $\phi_{1}$ and $\phi_{2}$ in (1) can be replaced by $\pi / 2+m \cdot \sum_{i} \cos \left(\Omega_{i} t\right)$ and $m \cdot \sum_{i} \sin \left(\Omega_{i} t\right)$, respectively, where $\Omega_{i}$ is the radian carrier frequency of the subcarrier channel $i$, and all channels have the same rms OMI value $m$. Fig. 3 shows the optical spectrum of a 2-ch SSB/SCM signal. The resultant SSB/SCM-modulation third-order nonlinear distortions (NLDs) are summarized in Table I.
It is well known that when the number of subcarrier channels is large, the dominant third-order NLD is the triple-beat product, which is expressed in decibels as

$$
\mathrm{CTB}_{\mathrm{SSB} / \mathrm{SCM}, \mathrm{MZI}}=10 \cdot \log \left\{4 \cdot\left[\frac{J_{1}(m)}{J_{0}(m)}\right]^{4} \cdot N_{\mathrm{CTB}}\right\}
$$

where $N_{\text {Ств }}$ is the triple-beat product count at a particular subcarrier channel of interest, whose center frequency $\Omega_{d}$ is equal to $\Omega_{i} \pm \Omega_{j} \pm \Omega_{k}$.

\section{B. Composite Second-Order Due to Linear Fiber Dispersion}

The derivations in Section III-B can be extended to the case of multiple subcarrier channels, and we summarize the results of various second-order NLDs in a transmission system using conventional single-mode fiber with a length of $z$ in Table II.

The second-order intermodulation distortion terms include two categories $\mathrm{IM}_{i+j}$ and $\mathrm{IM}_{i-j}$ as a result of SSB/SCM modulation. The reason why the amplitudes of $\mathrm{IM}_{i+j}$ and $\mathrm{IM}_{i-j}$ are different can be explained as follows. $\mathrm{IM}_{i+j}$ results from two types of beatings, i.e., $\omega_{0}$ beats with $\left[\omega_{o}+\left(\Omega_{1}+\Omega_{2}\right)\right]$ or $\left[\omega_{o}-\left(\Omega_{1}+\Omega_{2}\right)\right]$, while $\mathrm{IM}_{i-j}$ results from three types of beatings, i.e., in addition to $\omega_{0}$ beating with $\left[\omega_{o}+\left(\Omega_{1}-\Omega_{2}\right)\right]$ or $\left[\omega_{o}-\left(\Omega_{1}-\Omega_{2}\right)\right]$, there is a third nonnegligible contributing term from the beating between $\left(\omega_{o}+\Omega_{1}\right)$ and $\left(\omega_{o}+\Omega_{2}\right)$. This is because the second-order beating term $\pm\left[\left(\omega_{0}+\Omega_{2}\right)-\left(\omega_{0}+\Omega_{1}\right)\right]$ occurs in band, while the other second-order beating term $\left[\left(\omega_{o}+\Omega_{2}\right)+\left(\omega_{o}+\Omega_{1}\right)\right]$ occurs out of band.

Note that in an optical double-sideband system, the contributing terms to $\mathrm{IM}_{i+j}$ and $\mathrm{IM}_{i-j}$ are equal, because there are signal channels $\omega_{0}-\Omega_{1}$ and $\omega_{0}-\Omega_{2}$ at the other side of the optical carrier so that $\left[\left(\omega_{o}+\Omega_{2}\right)-\left(\omega_{o}-\Omega_{1}\right)\right]=\Omega_{2}+\Omega_{1}$ or $\left[\left(\omega_{o}+\Omega_{1}\right)-\left(\omega_{o}-\Omega_{2}\right)\right]=\Omega_{1}+\Omega_{2}$ are also in band.

Note that $\mathrm{IM}_{i-j}$ in Table II is not only distortion frequency $\left(\Omega_{d}\right)$ dependent, but also source frequency $\left(\Omega_{i}\right.$ and $\left.\Omega_{j}\right)$ dependent.

When the number of subcarrier channels is large, the dominant second-order NLD is the combination of $\mathrm{IM}_{i+j}$ and $\mathrm{IM}_{i-j}$ and is expressed in decibels as

$$
\begin{aligned}
& \mathrm{CSO}_{\mathrm{SSB} / \mathrm{SCM}, D} \\
& =10 \cdot \log \left\{\mathrm{IM}_{i+j} \cdot N_{\Omega_{i}+\Omega_{j}=\Omega_{d}}+\sum_{\Omega_{i}-\Omega_{j}=\Omega_{d}} \mathrm{IM}_{i-j}\right\}
\end{aligned}
$$

where $\Omega_{i}$ and $\Omega_{j}$ should be arbitrarily chosen from the frequencies of multiple subcarrier channels and $N_{\Omega_{i}+\Omega_{j}=\Omega_{d}}$ is the

$$
\begin{aligned}
I=\Re \cdot \eta \cdot\left|E_{\text {out }, D}\right|^{2} \approx \frac{\Re \eta\left|E_{\text {in }}\right|^{2}}{2}\{ & J_{0}^{2}(m)-2 \sqrt{2} J_{0}(m) J_{1}(m) \cdot \cos \left(\Omega t-\beta_{1} \Omega z-\frac{1}{2} \beta_{2} \Omega^{2} z-\frac{\pi}{4}\right) \\
& -4 J_{0}(m) J_{2}(m) \sin \left(\frac{1}{2} \beta_{2}(2 \Omega)^{2} z\right) \cdot \cos \left(2 \Omega t-\beta_{1}(2 \Omega) z\right) \\
& +2 \sqrt{2} C_{1} \cdot \cos \left(3 \Omega t-\beta_{1}(3 \Omega) z+\frac{\pi}{4}\right) \\
& \left.-2 \sqrt{2} C_{2} \cdot \sin \left(3 \Omega t-\beta_{1}(3 \Omega) z+\frac{\pi}{4}\right)+\cdots\right\}
\end{aligned}
$$


TABLE I

ANALYTIC EXPRESSIONS FOR THE INDIVIDUAL THIRD-ORDER DISTORTIONS DUE TO SSB/SCM Modulation. $\Omega_{i}, \Omega_{j}$, AND $\Omega_{k}$ ARE THreE ARBITRARILY CHOSEN Distinct SubCARrier FreQuENCIES

\begin{tabular}{c|c|c}
\hline Distortion Type & $\begin{array}{c}\text { Distortion Frequency } \\
\left(\Omega_{d}\right)\end{array}$ & Formula \\
\hline Third Harmonic & $3 \Omega_{i}$ & {$\left[\frac{J_{0}(m) J_{3}(m)-J_{1}(m) J_{2}(m)}{J_{0}(m) J_{1}(m)}\right]^{2}$} \\
\hline Third-Order Intermodulation & $2 \Omega_{i} \pm \Omega_{j}$ & {$\left[\frac{J_{1}(m)}{J_{0}(m)}\right]^{4}$} \\
\hline Triple Beat & $\Omega_{i} \pm \Omega_{j} \pm \Omega_{k}$ & $4 \cdot\left[\frac{J_{1}(m)}{J_{0}(m)}\right]^{4}$ \\
\hline
\end{tabular}

TABLE II

ANALYTIC EXPRESSIONS FOR SSB/SCM SECOND-ORDER NLDs DUE TO LINEAR FIBER DISPERSION. $\rho=1 / 2 \cdot \beta_{2} \cdot z$

\begin{tabular}{c|c|c}
\hline Distortion Type & Distortion Frequency $\left(\Omega_{d}\right)$ & Formula \\
\hline Second Harmonic & $2 \Omega_{i}$ & $2\left[\frac{J_{2}(m) \sin \left(\rho \cdot \Omega_{d}{ }^{2}\right)}{J_{1}(m)}\right]^{2}$ \\
\hline $2^{\text {nd }}$ order Intermodulation & $\Omega_{i}+\Omega_{j}$ & $2\left[\frac{J_{1}(m) \sin \left(\rho \cdot \Omega_{d}{ }^{2}\right)}{J_{0}(m)}\right]^{2}$ \\
$\left(I M_{i+j}\right)$ & $\Omega_{i}-\Omega_{j}$ & $2 \cdot\left[\frac{J_{1}(m)}{J_{0}(m)}\right]^{2} \cdot C^{*}$ \\
\hline $2^{\text {nd }}$ order Intermodulation & & \\
\hline
\end{tabular}

${ }^{*} C=\left[\cos \left(\rho \cdot\left(\Omega_{i}{ }^{2}-\Omega_{j}{ }^{2}\right)\right)-\cos \left(\rho \cdot \Omega_{d}{ }^{2}\right)\right]^{2}+\left[\sin \left(\rho \cdot\left(\Omega_{i}{ }^{2}-\Omega_{j}{ }^{2}\right)\right)\right]^{2}$

number of second-order intermodulation components whose source beating frequencies satisfy $\Omega_{i}+\Omega_{j}=\Omega_{d}$. On the other hand, when the distortion is source beating frequency dependent, the accumulation of the second-order intermodulation distortions should be written as the summation form such as the second term in (12).

\section{C. СТВ Due to Linear Fiber Dispersion}

The derivations on linear-fiber-dispersion-induced harmonic distortions in Section III-B can be extended to third-order intermodulation distortions as

$$
\mathrm{IM}_{i \pm j \pm k}=\left[\frac{J_{1}(m)}{J_{0}(m)}\right]^{4} \cdot\left(C 1_{i \pm j \pm k}^{2}+C 2_{i \pm j \pm k}^{2}\right)
$$

where $C 1_{i \pm j \pm k}$ and $C 2_{i \pm j \pm k}$ are given by (note that $\rho$ in these equations is again given by $\left.1 / 2 \cdot \beta_{2} \cdot z\right)$

$$
\begin{aligned}
& C 1_{i+j+k} \\
= & \cos \left[\rho \cdot\left(\Omega_{i}+\Omega_{j}+\Omega_{k}\right)^{2}\right]-\cos \left[\rho \cdot\left(\Omega_{i}^{2}-\left(\Omega_{j}+\Omega_{k}\right)^{2}\right)\right] \\
& -\cos \left[\rho \cdot\left(\Omega_{j}^{2}-\left(\Omega_{i}+\Omega_{k}\right)^{2}\right)\right]-\cos \left[\rho \cdot\left(\Omega_{k}^{2}-\left(\Omega_{i}+\Omega_{j}\right)^{2}\right)\right]
\end{aligned}
$$

$$
C 2_{i+j+k}
$$$$
=\sin \left[\rho \cdot\left(\Omega_{i}+\Omega_{j}+\Omega_{k}\right)^{2}\right]+\sin \left[\rho \cdot\left(\Omega_{i}^{2}-\left(\Omega_{j}+\Omega_{k}\right)^{2}\right)\right]
$$$$
+\sin \left[\rho \cdot\left(\Omega_{j}^{2}-\left(\Omega_{i}+\Omega_{k}\right)^{2}\right)\right]+\sin \left[\rho \cdot\left(\Omega_{k}^{2}-\left(\Omega_{i}+\Omega_{j}\right)^{2}\right)\right]
$$

$$
\begin{aligned}
& C 1_{i+j-k} \\
= & -\cos \left[\rho \cdot\left(\Omega_{i}+\Omega_{j}-\Omega_{k}\right)^{2}\right]-\cos \left[\rho \cdot\left(\Omega_{i}^{2}-\left(\Omega_{j}-\Omega_{k}\right)^{2}\right)\right] \\
& -\cos \left[\rho \cdot\left(\Omega_{j}^{2}-\left(\Omega_{i}-\Omega_{k}\right)^{2}\right)\right]+\cos \left[\rho \cdot\left(\Omega_{k}^{2}-\left(\Omega_{i}+\Omega_{j}\right)^{2}\right)\right] \\
& C 2_{i+j-k} \\
= & \sin \left[\rho \cdot\left(\Omega_{i}+\Omega_{j}-\Omega_{k}\right)^{2}\right]+\sin \left[\rho \cdot\left(\Omega_{i}^{2}-\left(\Omega_{j}-\Omega_{k}\right)^{2}\right)\right] \\
& +\sin \left[\rho \cdot\left(\Omega_{j}^{2}-\left(\Omega_{i}-\Omega_{k}\right)^{2}\right)\right]+\sin \left[\rho \cdot\left(\Omega_{k}^{2}-\left(\Omega_{i}+\Omega_{j}\right)^{2}\right)\right] \\
& C 1_{i-j+k} \quad(14 \mathrm{e}) \\
= & -\cos \left[\rho \cdot\left(\Omega_{i}-\Omega_{j}+\Omega_{k}\right)^{2}\right]-\cos \left[\rho \cdot\left(\Omega_{i}^{2}-\left(-\Omega_{j}+\Omega_{k}\right)^{2}\right)\right] \\
& +\cos \left[\rho \cdot\left(\Omega_{j}^{2}-\left(\Omega_{i}+\Omega_{k}\right)^{2}\right)\right]-\cos \left[\rho \cdot\left(\Omega_{k}^{2}-\left(\Omega_{i}-\Omega_{j}\right)^{2}\right)\right] \\
& C 2_{i-j+k} \\
= & \sin \left[\rho \cdot\left(\Omega_{i}-\Omega_{j}+\Omega_{k}\right)^{2}\right]+\sin \left[\rho \cdot\left(\Omega_{i}^{2}-\left(-\Omega_{j}+\Omega_{k}\right)^{2}\right)\right] \\
& +\sin \left[\rho \cdot\left(\Omega_{j}^{2}-\left(\Omega_{i}+\Omega_{k}\right)^{2}\right)\right]+\sin \left[\rho \cdot\left(\Omega_{k}^{2}-\left(\Omega_{i}-\Omega_{j}\right)^{2}\right)\right] \\
& C 1_{i-j-k} \\
= & \cos \left[\rho \cdot\left(\Omega_{i}-\Omega_{j}-\Omega_{k}\right)^{2}\right]-\cos \left[\rho \cdot\left(\Omega_{i}^{2}-\left(-\Omega_{j}-\Omega_{k}\right)^{2}\right)\right] \\
& +\cos \left[\rho \cdot\left(\Omega_{j}^{2}-\left(\Omega_{i}-\Omega_{k}\right)^{2}\right)\right]+\cos \left[\rho \cdot\left(\Omega_{k}^{2}-\left(\Omega_{i}-\Omega_{j}\right)^{2}\right)\right] \\
& C 2_{i-j-k}(14 \mathrm{~g}) \\
= & \sin \left[\rho \cdot\left(\Omega_{i}-\Omega_{j}-\Omega_{k}\right)^{2}\right]+\sin \left[\rho \cdot\left(\Omega_{i}^{2}-\left(-\Omega_{j}-\Omega_{k}\right)^{2}\right)\right] \\
& +\sin \left[\rho \cdot\left(\Omega_{j}^{2}-\left(\Omega_{i}-\Omega_{k}\right)^{2}\right)\right]+\sin \left[\rho \cdot\left(\Omega_{k}^{2}-\left(\Omega_{i}-\Omega_{j}\right)^{2}\right)\right] .
\end{aligned}
$$


We can write the CTB in decibels as

$$
\mathrm{CTB}_{\mathrm{SSB} / \mathrm{SCM}, D}=10 \cdot \log \left(\sum_{\Omega_{i} \pm \Omega_{j} \pm \Omega_{k}=\Omega_{d}} \mathrm{IM}_{i \pm j \pm k}\right)
$$

where $\Omega_{i}, \Omega_{j}$, and $\Omega_{k}$ could be chosen arbitrarily from the frequencies of multiple subcarrier channels, and $\Omega_{d}$ is the distortion frequency.

\section{Theoretical Predictions and Simulation Results}

Computer simulations based on the Photonic Transmission Design Suite (PTDS) (a physical layer design tool developed by Virtual Photonics, Inc.) were carried out to verify the analytical results derived in Sections IV-A to IV-C, and the results are given hereafter. Note that we used a time window (the reciprocal of "frequency resolution") of 128/155e6 s, and a sample rate of $8 \times 64 \times 155 \mathrm{e} 6 \mathrm{~Hz}(79.36 \mathrm{GHz})$.

To verify the results derived in Sections IV-A and IV-B, we used 64 channels of quadrature phase-shift-keying (QPSK) signals in the frequency range $0.93-12.648 \mathrm{GHz}$. The rms OMI per channel was assumed to be $4 \%$. Each QPSK signal has a symbol rate of $155 \mathrm{Msymbol} / \mathrm{s}$ and an excess bandwidth factor of 0.2 , and the channel spacing is $186 \mathrm{MHz}(1.2 \times 155 \mathrm{MHz})$. Bit streams among those 64 channels were assumed to be uncorrelated.

To study the fundamental nonlinear distortions due to the intrinsic Mach-Zehnder modulator nonlinearity and fiber dispersion, thermal and shot noise are not taken into account. At the receiver end, the noise-to-signal ratio (NSR) is measured to estimate the impact of NLDs. The reason why we use the NSR (instead of the signal-to-noise ratio, or SNR) is because CSO/CTBs are noise-like in multichannel digital modulation SCM systems; therefore, NSR and CSO/CTB are essentially equivalent [5]. Continuous-wave $(\mathrm{CW}) \mathrm{RF} /$ microwave tones are used to represent the modulated signals [5].

Two cases were studied: back-to-back and 80-km SMF transmission (with a fiber dispersion of $17 \mathrm{ps} / \mathrm{nm} / \mathrm{km}$ at $1.55 \mu \mathrm{m}$ ). In both cases, after all subcarrier channels were converted to electrical signals by a photodiode, and one of the 64 channels was sampled and electrically filtered to measure its NSR performance. The sampled channel should be turned off while measuring the total noise-like NLD in its bandwidth. Since different orders of NLD falling into a particular channel cannot be separated in the simulation, we need to know the dominant NLD in each case-for the case of back-to-back, the dominant NLD is CTB due to the MZI modulator, and that is the fundamental system limit after all fiber dispersions are compensated. For the case of an 80-km transmission, both CSO and CTB should be considered, although the dominant NLD is CSO. This is because that CSO is proportional to $m^{2}$, while CTB is proportional to $m^{4}$, as can be observed from (11) and (12) by letting $J_{0}(m) \approx 1, J_{1}(m) \approx m, J_{2}(m) \approx m^{2} / 2$, and $J_{3}(m) \propto m^{3}$ for $\mathrm{rms} \mathrm{OMI} / \mathrm{ch} \ll 1$. Therefore, in a multichannel, dispersive system with a small rms OMI per channel, CSOs are the dominant nonlinear terms.

Fig. 4 shows the frequency distribution of the resultant NSR for transmission lengths of 0 and $80 \mathrm{~km}$, respectively. In the case of $0 \mathrm{~km}$, the simulation result agrees well with the analytical

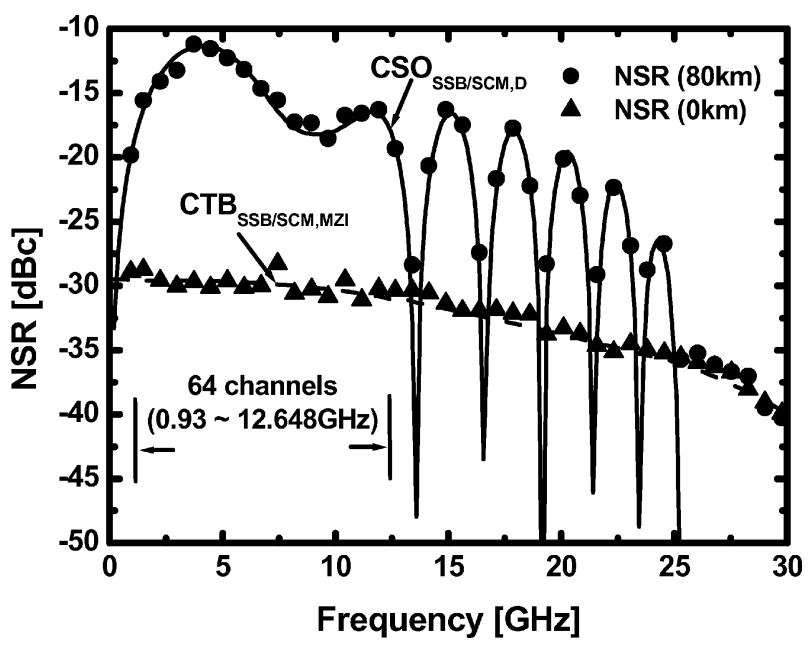

Fig. 4. NSR versus frequency in an SSB/SCM optical transmission system with 64 QPSK channels (ranging from 0.93 to $12.648 \mathrm{GHz}$ ), for fiber lengths of 0 and $80 \mathrm{~km}$. Dashed line and upper triangular symbols are the theoretical and simulation results for the case of no fiber transmission, respectively. Solid lines and circular symbols are the theoretical and simulation results after $80-\mathrm{km}$ fiber transmission, respectively. The rms OMI per channel is around $4 \%$.

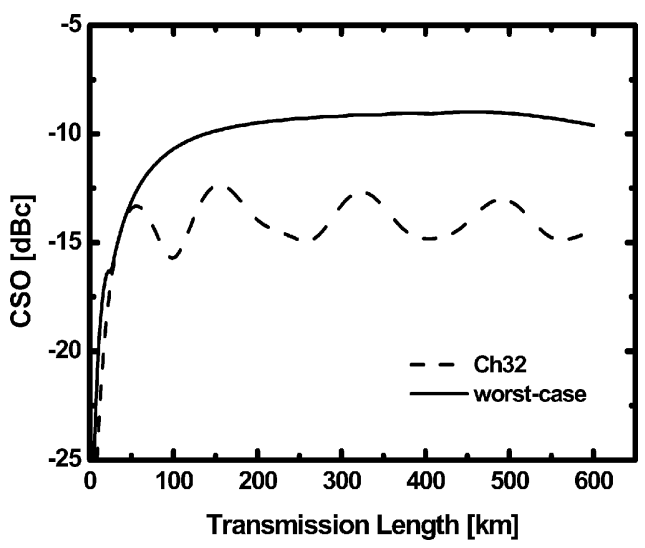

Fig. 5. Linear-fiber-dispersion-induced CSO versus transmission distance in a SCM system with the 64 QPSK channels shown in Fig. 4. Dashed and solid lines are the results of channel 32 and the worst-case channel, respectively. The rms OMI per channel is $4 \%$.

CTB approximation obtained from (11). However, in the case of $80-\mathrm{km}$ SMF fiber transmission, although the simulation result agrees quite well with the analytical CSO approximation obtained from (12) in most cases, there does exist some large discrepancy at those frequencies close to dips. This is because at those dips, CTBs due to linear fiber dispersion are not negligible.

Fig. 5 shows the linear-fiber-dispersion-induced CSO as a function of transmission distance. At an OMI per channel of $4 \%$, the CSO in channel 32 (dashed line) first monotonously increases with transmission distance until at about $50 \mathrm{~km}$ and then fluctuates around $\sim-14 \mathrm{~dB}$ with a peak-to-peak variation of $\sim 2.5 \mathrm{~dB}$. This phenomenon may be explained from $\mathrm{IM}_{i-j}$ in (12), where each contribution has distance-dependent cosine or sine terms and with different period along the transmission distance $z$. The solid line in Fig. 5 represents the CSO in a worst-case channel at different distances. Note that the worst-case channel is not a fixed channel; rather, it is distance dependent, i.e., for each distance, the worst CSO may occur 


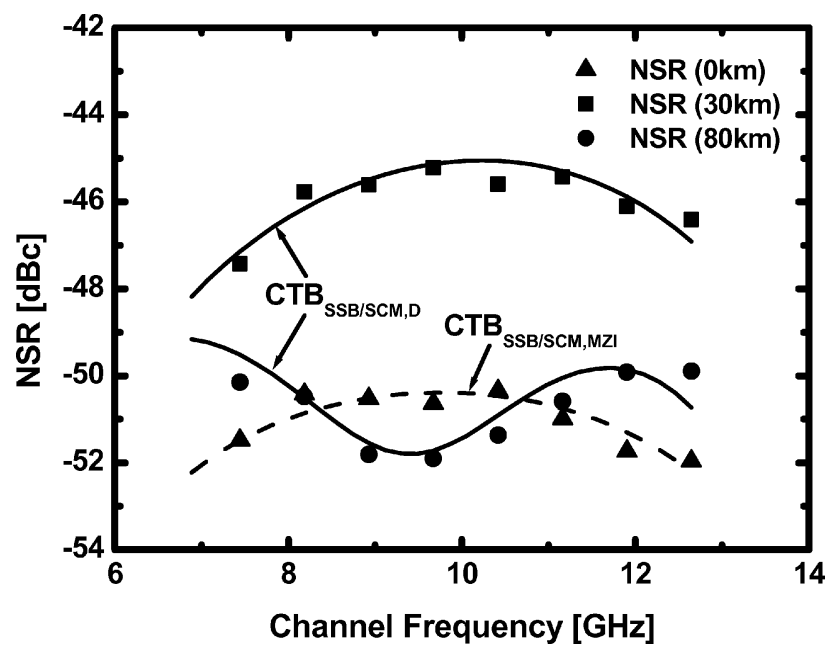

Fig. 6. NSR versus frequency in an SSB/SCM optical transmission system with 32 16-QAM channels (ranging from 6.882 to $12.648 \mathrm{GHz}$ ) for fiber lengths of 0,30 , and $80 \mathrm{~km}$. Dashed line and upper triangular symbols are the theoretical and simulation results for the case of no fiber transmission, respectively. Square and circle symbols are the simulation results after 30- and $80-\mathrm{km}$ fiber transmissions, respectively. Solid lines are the theoretical results. The rms OMI per channel is around $1.8 \%$.

in different channels. It can be seen that the worst-case CSO increases with distance initially but saturates at $\sim-10 \mathrm{~dB}$ beyond about $100 \mathrm{~km}$.

It is of interest to compare SSB/SCM system to a conventional SCM system in which two sidebands exist on each side of an optical carrier. The transmission distance of the latter is known to be bounded by the dispersion-limited bandwidth [6], rather than by CSOs. The limited transmission bandwidth is caused by the periodic cancellation of the two sidebands, as we mentioned in Section I. For example, when a conventional SCM system has the same maximum microwave subcarrier frequency of $12.648 \mathrm{GHz}$ as in the current $\mathrm{SSB} / \mathrm{SCM}$ system, its transmission distance is limited to only $\sim 23 \mathrm{~km}$.

To verify the results derived in Section IV-C, a computer simulation on fiber-dispersion-induced CTB was carried out for the case of 32 16-quadrature amplitude modulation (16-QAM) signals between 6.882 and $12.648 \mathrm{GHz}$ (within an octave), with an rms OMI per channel of $1.8 \%$. Fig. 6 shows the frequency distribution of the calculated and simulated NSR for transmission lengths of 0,30 , and $80 \mathrm{~km}$, respectively. In the case of $0 \mathrm{~km}$, the dominant NLD is the intrinsic MZI-induced CTB (see (11)). However, with fiber transmissions, the dominant NLD becomes the fiber-dispersion-induced CTB. It is clear that the simulation results agree well with the analytical CTB given in (15). Note the dispersion-induced CTB at $30 \mathrm{~km}$ is more severe than that at $80 \mathrm{~km}$. This is because the dispersion-induced CTBs oscillate around the MZI-induced CTB, as shown in Fig. 7 and is due to the cosine and sine terms in (14a)-(14h).

Fig. 7 shows the linear-fiber-dispersion-induced CTB as a function of transmission distance. The intrinsic MZI-induced CTB of channel 16 (dotted line) obtained from (11) is shown as a distance-independent baseline. It is shown that the CTB in channel 16 (dashed line) irregularly oscillates around the baseline, and the deviation from the baseline becomes smaller as the

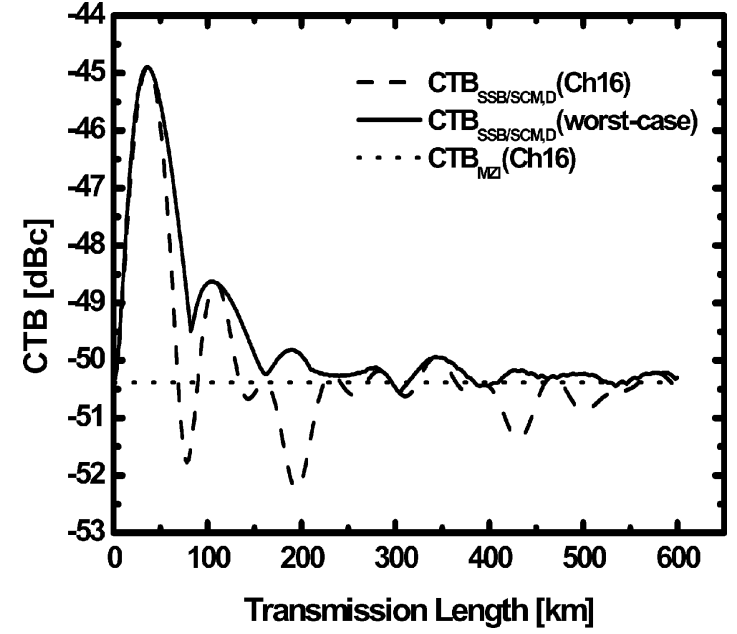

Fig. 7. Linear-fiber-dispersion-induced CTB versus transmission distance in an SSB/SCM system with 32 16-QAM channels. Dashed and solid lines are the results of channel 16 and the worst-case channel, respectively. Dotted line is the intrinsic MZI-induced CTB at channel 16. The rms OMI per channel is $1.8 \%$.

distance increases. This may be attributed to the distance-dependent cosine and sine terms in (14a)-(14h). Note that the worst-case CTB, which occurs in channel 16 , has a maximum value of $\sim-45 \mathrm{~dB}$ after a fiber transmission of $\sim 30 \mathrm{~km}$. Furthermore, the worst-case CTB approaches the baseline beyond a certain distance, i.e., $150 \mathrm{~km}$ in the current QAM/SSB/SCM system. This result has an important implication to future metro and wireless optical networks in that the transmission distance of a high-capacity SSB/SCM system can be increased significantly, provided that all SCM channels can be kept within one octave.

\section{SPM-INDUCED IMPAIRMENT}

The effects of SPM in an SSB/SCM system include the following:

1) SPM-induced subcarrier amplitude fluctuation, which varies among multiple modulation frequencies (considering a fixed distance);

2) SPM and chromatic-dispersion-induced residual power on the other optical sideband [7];

3) SPM-induced nonlinear distortions.

We have found that the third factor is negligible when compared with the nonlinear distortions caused by linear fiber dispersion and nonlinear XPM (in the case of multiple wavelengths). The first and second factors can also be ignored if we limit the launched power per wavelength to below $\sim 6 \mathrm{dBm}$, which gives a subcarrier-to-subcarrier amplitude variation of less than $1 \mathrm{~dB}$ [7], [8].

\section{DWDM CONFIGURATION-XPM LIMITATIONS}

XPM generates signal phase noise, which may be converted to amplitude noise through dispersion. The dispersion can come from the fiber plant or any other dispersive optical elements such as filters or DWDM demultiplexers [9]. The latter is not considered in this paper, i.e., we assume that the transmission slope in the passband of a receiving filter or demultiplexer is negligible. In this section, the effect of XPM on SSB/SCM/DWDM optical 
fiber links is investigated theoretically and numerically. A generalized theoretical model of crosstalk (XT) induced by an arbitrary number of wavelength channels through XPM is derived and is verified by computer simulations.

\section{A. XPM-Induced XT Considering Two Wavelengths}

Here, we generalize the analysis presented in [10] and [11] to obtain the results for SSB/SCM systems. Note that the smallsignal perturbation approach shown in [4] cannot be applied to subcarrier frequencies higher than about $1 \mathrm{GHz}$ and is not applicable to the microwave SSB/SCM system under consideration. We first consider a $\mathrm{CW}$ probe wavelength and a modulated pump wavelength with the same polarization copropagating in a single-mode optical fiber. At the fiber input, the expression for a sine-modulated pump channel is given by

$$
\widetilde{P}_{p}(t)=m \bar{P}_{p}(0) \cdot \cos (\Omega t+\theta), \text { (input pump) }
$$

whose complex amplitude is given by $\widetilde{P}_{p}(\Omega)=m \bar{P}_{p}(0) \cdot e^{j \theta}$, where $\bar{P}_{p}(0)$ is the average pump power at the fiber input. The change of the pump signal power due to both chromatic dispersion and fiber nonlinearity can modulate the phase of a probe channel through XPM. Let us assume the normalized complex amplitude of the pump signal at a distance $z$ can be expressed as [11]

$\tilde{P}_{p}(z, \Omega)=\tilde{P}_{p}(\Omega) \cdot H_{F p}(z, \Omega) \cdot e^{-\alpha z} e^{-j \Omega z / v_{g p}}$

(pump at a distance $z$ )

where $e^{-\alpha z} e^{-j \Omega z / v_{g p}}$ accounts for the attenuation and propagation delay at a distance $z$ from the fiber input. Note that $v_{g p}$ and $\alpha$ are the group velocity and attenuation coefficient, respectively. The function $H_{F_{x}}(z, \Omega)(x=\mathrm{p}$ for a pump channel, and $x=\mathrm{b}$ for a probe channel) is defined as the ratio between the intensity modulation at the fiber output (at a distance $z$ ) and fiber input $(z=0)$ [12] and is called the intensity modulation-fiber transfer function (IM-FTF), which takes into account the interaction of fiber nonlinearity and dispersion. To separate the effect of IM-IM and phase modulation (PM)-IM conversions, we can express $H_{F_{x}}(z, \Omega)$ as [12]-[17]

$$
H_{F_{x}}(z, \Omega)=C_{\mathrm{IM}-\mathrm{IM}}(z, \Omega)+H_{\mathrm{PM}}(\Omega) \cdot C_{\mathrm{PM}-\mathrm{IM}}(z, \Omega)
$$

where $C_{\mathrm{IM}-\mathrm{IM}}(z, \Omega)=\widetilde{p}_{N}(z, \Omega) /\left.\widetilde{p}_{N}(0, \Omega)\right|_{\tilde{\phi}(0, \Omega)=0}$ is a conversion function representing IM-IM conversion, $C_{\mathrm{PM}-\mathrm{IM}}(z, \Omega)=\tilde{p}_{N}(z, \Omega) /\left.[2 \bar{P}(0) \cdot \tilde{\phi}(0, \Omega)]\right|_{\tilde{p}_{N}(0, \Omega)=0}$ is a conversion function representing PM-IM conversion, and $H_{\mathrm{PM}}(\Omega)=2 \bar{P}(0) \cdot \widetilde{\phi}(0, \Omega) / \widetilde{p}_{N}(0, \Omega)$ gives the relation between IM and PM for a given optical transmitter. Note that $\widetilde{p}_{N}(z, \Omega)$ and $\widetilde{\phi}(z, \Omega)$ are the Fourier transforms of the normalized signal power and phase terms $\widetilde{p}_{N}(z, t)$ and $\widetilde{\phi}(z, t)$ (normalized by $\bar{P}(0)$ ) of the pump channel (or probe channel), respectively.

In a directly modulated semiconductor laser [13]-[15], $H_{\mathrm{PM}}(\Omega)=\alpha_{c}\left(1-j f_{c} / f\right)$, where $\alpha_{c}$ is the chirp parameter and $f_{c}$ is the frequency at which the adiabatic chirp equals to the transient chirp. In an externally modulated IM-direct detection (DD) system [17], $H_{\mathrm{PM}}(\Omega)=\alpha_{c}$. In an SSB/SCM modulation system with ideal dual-electrode MZI modulator [7], i.e., the case we are interested in, $H_{\mathrm{PM}}(\Omega)= \pm j(+j$ represents the optical lower sideband (LSB), whereas $-j$ represents the optical upper sideband (USB)).

For a given $H_{\mathrm{PM}}(\Omega)$, one can numerically solve $C_{\mathrm{IM}-\mathrm{IM}}(z, \Omega), C_{\mathrm{PM}-\mathrm{IM}}(z, \Omega)$, and $H_{F_{x}}(z, \Omega)$ (by using the fourth-fifth-order Runge-Kutta method) from the following coupled linear differential equations, which depicts the evolution of small-signal power $\widetilde{p}_{N}(z, \Omega)$ and phase $\widetilde{\phi}(z, \Omega)$ along a transmission link by considering both GVD and fiber nonlinearities [18], as follows:

$$
\begin{aligned}
\frac{\partial \widetilde{p}_{N}(z, \Omega)}{\partial z} & =\beta_{2} \Omega^{2} \bar{P}(0) \cdot \widetilde{\phi}(z, \Omega) \\
\frac{\partial \widetilde{\phi}(z, \Omega)}{\partial z} & =-\left[\frac{\beta_{2} \Omega^{2}}{4 \bar{P}(0)}+\gamma \cdot \exp (-\alpha z)\right] \cdot \widetilde{p}_{N}(z, \Omega)(1
\end{aligned}
$$

where $\beta_{2}$ is the first-order GVD parameter presented in Section III, $\gamma=2 \pi n_{2} /\left(\lambda A_{\text {eff }}\right)$ is the nonlinearity coefficient accounting for the nonlinear Kerr effect, $n_{2}$ is the nonlinear refractive index, and $A_{\text {eff }}$ the effective core area of the optical fiber.

Once $H_{F p}(z, \Omega)$ is obtained, the XPM-induced complex sinusoidal PM in the probe channel (originated in an infinitesimal section of length $d z$ at a distance $z$ from the fiber input) is given by [11]

$$
\begin{aligned}
& d \tilde{\phi}_{\mathrm{XPM}}(z, \Omega) \\
& \quad=-2 \gamma_{b} \tilde{P}_{p}(\Omega) \cdot H_{F p}(z, \Omega) \cdot e^{-\alpha z} e^{-j \Omega z / v_{g p}} d z
\end{aligned}
$$

(XPM-induced PM in a probe channel)

where $\gamma_{b}$ is the nonlinear coupling coefficient of a probe channel.

XPM-induced PM in a probe channel is converted to IM at the output of a transmission link through PM-IM conversion. The total XPM-induced IM of a probe channel at the output of a transmission link with a length $L$ is given by (19), shown at the bottom of the page, where $\bar{P}_{b}(z)=\bar{P}_{b}(0) \cdot e^{-\alpha z}$ is the average power of the probe channel at a distance $z$, and $\bar{P}_{b}(0)$ is the average power of a probe channel at the fiber input; $e^{-\alpha(L-z)} e^{-j \Omega(L-z) / v_{g b}}$ represents the attenuation and propagation delay of the probe channel from $z$ to $L$; and $C_{\mathrm{PM}-\mathrm{IM}, b}(L-z, \Omega)$ is the PM-IM conversion of the probe channel from $z$ to $L$.

$$
\widetilde{P}_{\mathrm{XPM}}(z, \Omega)=\int_{0}^{L} 2 \bar{P}_{b}(z) \cdot e^{-\alpha(L-z)} e^{-j \Omega(L-z) / v_{g b}} \cdot C_{\mathrm{PM}-\mathrm{IM}, b}(L-z, \Omega) \cdot d \widetilde{\phi}_{\mathrm{XPM}}(z, \Omega)
$$

(PM-to-IM induced IM in a probe channel) 
Substituting (18) into (19) yields

$$
\begin{aligned}
& \tilde{P}_{\mathrm{XPM}}(\Omega)=-4 \gamma_{b} \bar{P}_{b}(0) \cdot \tilde{P}_{p}(\Omega) \cdot e^{-\alpha L} e^{-j \Omega L / v_{g b}} \\
& \quad \cdot \int_{0}^{L} \exp (-\sigma z) \cdot H_{F p}(z, \Omega) \cdot C_{\mathrm{PM}-\mathrm{IM}, b}(L-z, \Omega) \cdot d z
\end{aligned}
$$

where $\sigma=\alpha-j \Omega d_{b p}$ and $d_{b p} \equiv\left(v_{g b}\right)^{-1}-\left(v_{g p}\right)^{-1}=$ $\int_{\lambda_{p}}^{\lambda_{b}} D(\lambda) d \lambda$ is the walkoff parameter between pump and probe wavelengths. In a nonzero dispersion region, $d_{b p} \approx D_{b} \cdot \Delta \lambda_{b p}-$ $S_{b} \cdot\left(\Delta \lambda_{b p}\right)^{2}$, where $\Delta \lambda_{b p} \equiv \lambda_{b}-\lambda_{p}$ is the separation between probe and pump wavelengths, and $S_{b} \equiv d D /\left.d \lambda\right|_{\lambda_{b}}$ is the dispersion slope of the probe channel.

Note that $H_{F p}(z, \Omega)$ in (20), instead of being numerically obtained via (16) and (17), can possibly be approximated by using a closed-form obtained from a linear dispersive system. In the next section, we will point out the limitation of this approach, despite that it could save significant computing time and help us gain more physical insights. The IM-IM and PM-IM conversions of a pump channel can be expressed by $[12] C_{\mathrm{IM}-\mathrm{IM}, p}(z, \Omega)=\cos \left(\Theta_{p} z\right)$ and $C_{\mathrm{PM}-\mathrm{IM}, p}(z, \Omega)=-\sin \left(\Theta_{p} z\right)$, respectively, where $\Theta_{p}=\Omega^{2} D_{p} \lambda_{p}^{2} /(4 \pi c)$ with $D_{p}$ being the dispersion coefficient of the pump channel. Consequently, the IM-FTF of a pump channel is given by a simplified expression: $H_{F p}(z, \Omega)=\cos \left(\Theta_{p} z\right)-H_{\mathrm{PM}}(\Omega) \cdot \sin \left(\Theta_{p} z\right)$.

In a USB/SCM system, $H_{F p}(z, \Omega)=H_{F p, \mathrm{USB}}(z, \Omega)=$ $\cos \left(\Theta_{p} z\right)+j \cdot \sin \left(\Theta_{p} z\right)$ and $C_{\mathrm{PM}-\mathrm{IM}, b}(L-z, \Omega)=$ $-\sin \left(\Theta_{b} z\right)$ can be used in (20) to obtain a closed-form solution as

$$
\begin{aligned}
\tilde{P}_{\mathrm{XPM}, \mathrm{USB}}(\Omega)= & 4 \gamma_{b} \bar{P}_{b}(0) \cdot \tilde{P}_{p}(\Omega) \cdot e^{-\alpha L} e^{-j \Omega L / v_{g b}} \\
& \cdot\left\{\frac { 1 } { ( \sigma - j \Theta _ { p } ) ^ { 2 } + \Theta _ { b } ^ { 2 } } \left[\left(\sigma-j \Theta_{p}\right) \cdot \sin \left(\Theta_{b} L\right)\right.\right. \\
& \left.\left.-\Theta_{b} \cdot \cos \left(\Theta_{b} L\right)+\Theta_{b} \cdot e^{-\left(\sigma-j \Theta_{p}\right) L}\right]\right\} .
\end{aligned}
$$

For an LSB/SCM system, the result in (21) can be modified by replacing $j \Theta_{p}$ with $-j \Theta_{p}$. The XT induced by XPM in a probe channel is given by $\mathrm{XT}_{\mathrm{XPM}}=$ $\left|\widetilde{P}_{\mathrm{XPM}}(\Omega) /\left(\widetilde{P}_{p}(\Omega) \cdot e^{-\alpha L}\right)\right|^{2}$,where $\widetilde{P}_{p}(\Omega)$ is the complex amplitude of a pump channel.

Equation (21) shows that the strength of the XPM-induced power fluctuation in a probe channel depends on the average power of the pump and probe channels, the modulation frequency $\Omega$, the walkoff, and dispersion parameters. Equation (21) also shows that in general the major contribution to the XPM-induced XT is generated in the front part of the fiber as a result of the optical power attenuation $e^{-\alpha L}$. Equation (21) further shows that only when there is a local zero chromatic dispersion at the probe wavelength (i.e., $\Theta_{b}=0$ ) can the XPM-induced XT be eliminated.

If we rewrite the term $\sigma-j \Theta_{p}$ in (21) as $\alpha-j\left(\Omega d_{b p} \pm \Theta_{p}\right)$, we can see that when $\Theta_{p}$ and the walkoff parameter $\Omega d_{b p}$ have the same sign, $\Theta_{p}$ increases the walkoff; whereas when they have different signs, $\Theta_{p}$ decreases the walkoff. It is interesting to see that the phase change effect due to GVD in SSB/SCM modulation is significantly comparable to the walkoff effect at a small channel separation and at a high modulation frequency. This can be easily observed from the ratio of $\Theta_{p}$ and $\Omega d_{b p}$, given by $\left(\Omega / \omega_{p}\right) /\left(2 \Delta \lambda_{b p} / \lambda_{p}\right)$, where $\omega_{p}$ is the optical angular frequency of the pump channel. For example, when the wavelength separation is $0.2 \mathrm{~nm}$ and the modulation frequency is 1 $\mathrm{GHz}$, the ratio of $\Theta_{p}$ and $\Omega d_{b p}$ is about 1/50, and therefore $\Theta_{p}$ is negligible. Following the same logic, this is also true when the channel spacing is more than $0.2 \mathrm{~nm}$. However, when the modulation frequency is increased to $>\sim 10 \mathrm{GHz}, \Theta_{p}$ becomes comparable to $\Omega d_{b p}$ and cannot be neglected.

From this discussion, it is possible to further simplify (21) by letting $\Theta_{p}=0$ when the wavelength-division-multiplexing (WDM) channel spacing is larger than $0.2 \mathrm{~nm}$ and when the $\mathrm{RF}$ modulation frequency is lower than a few $\mathrm{GHz}$. Under this condition, we have $H_{F p}(z, \Omega) \approx 1, \cos \left(\Theta_{b} L\right) \approx 1$, and $\sin \left(\Theta_{b} L\right) \approx \Omega^{2} D_{b} \lambda_{b}^{2} L /(4 \pi c)$. Consequently, XT based on (21) can be approximated as [4], [11]

$$
\begin{aligned}
X T_{\mathrm{XPM}}(\Omega)=\mid \gamma_{b} \bar{P}_{p}(0) & \cdot \frac{\lambda_{b}^{2}}{\pi c} D_{b} L \frac{\Omega^{2}}{\alpha-j \Omega d_{b p}} \\
\cdot & {\left.\left[1-\frac{1-e^{-\left(\alpha-j \Omega d_{b p}\right) L}}{\alpha-j \Omega d_{b p}}\right]\right|^{2} . }
\end{aligned}
$$

It can be seen from (22) that for a weak walkoff and small modulation frequencies $\left|\Omega d_{d p}\right| \ll \alpha$, the XPM-induced XT increases linearly with $\Omega^{4}$; while for a strong walkoff and higher modulation frequencies $\left|\Omega d_{d p}\right| \gg \alpha$, the XPM-induced XT is linearly proportional to $\Omega^{2}$ and inversely proportional to the square of channel spacing. Also note that XPM-induced XT is independent of OMI per channel.

Under the conditions of large wavelength spacing and small modulation frequencies, both an SSB/SCM system and a dispersion-compensated optical double sideband (ODSB) system have neither dispersion-induced fading nor pump envelope change, i.e., $\left|H_{F p}(z, \Omega)\right|=1$ (note that an ODSB system without dispersion compensation has $\left.H_{F p}(z, \Omega)=\cos \left(\Theta_{p} z\right)\right)$. It should be noted, however, that in an SSB/SCM system $H_{F p}(z, \Omega)=$ $\cos \left(\Theta_{p} z\right) \pm j \cdot \sin \left(\Theta_{p} z\right)=\exp \left( \pm j \Theta_{p} z\right)(+:$ USB; -: LSB); hence, $\left|H_{F p}(z, \Omega)\right|=1$ does not imply $\Theta_{p}=0$. Consequently, the assumption of $\Theta_{p}=0$ may cause errors, which we will discuss subsequently.

\section{B. Multiple Wavelengths}

Equation (18) can be easily extended to cases when there are more than two wavelengths copropagating in a fiber link. In those cases, each pump wavelength $k$ modulates the phase of a probe wavelength independently [19], [20], and therefore (18) can be modified as

$$
\begin{aligned}
& d \tilde{\phi}_{\mathrm{XPM}, \text { total }}(z, \Omega)= \\
& -2 \gamma_{b}\left[\sum_{k \neq b} \tilde{P}_{k}(\Omega) \cdot H_{F k}(z, \Omega) \cdot e^{-j \Omega z / v_{g k}}\right] \cdot e^{-\alpha z} d z
\end{aligned}
$$

where $\widetilde{P}_{k}(\Omega)$ and $H_{F k}(z, \Omega)$ are the complex amplitude and the IM-FTF of a pump channel $k$, respectively. 
TABLE III

PARAMETERS FOR 20-Gb/s SSB/SCM SYSTEMS

\begin{tabular}{l|l}
\hline Number of subcarrier channels on pump wavelength & 64 \\
\hline Bandwidth Efficient Modulation (BEM) scheme & QPSK \\
\hline Symbol rate per channel & $155 \mathrm{Msymbol} / \mathrm{s}$ \\
\hline Bandwidth excess factor & 0.2 \\
\hline Channel spacing & $1.2 \times 155 \mathrm{MHz}$ \\
\hline Channel frequency plan & $0.93 \sim 12.648 \mathrm{GHz}$ \\
\hline Optical modulation scheme & $\mathrm{USB} / \mathrm{SCM}$ \\
\hline Number of wavelengths & $2($ Fig.8), $4(\mathrm{Figs} .9-13)$ \\
\hline Wavelength separation & 25,50, and $100 \mathrm{GHz}$ \\
\hline Probe Wavelength $\left(\lambda_{3}\right)$ & $1550 \mathrm{~nm}$ \\
\hline Fiber dispersion coefficient at $\lambda_{3}(D)$ & $17 \mathrm{ps} / \mathrm{nm}^{\prime} / \mathrm{km}$ \\
\hline Fiber dispersion slope at $\lambda_{3}(S)$ & $0.044 \mathrm{ps} / \mathrm{nm}^{2} / \mathrm{km}$ \\
\hline Fiber nonlinear index coefficient $\left(n_{2}\right)$ & $2.6 \times 10^{-20} \mathrm{~m}^{2} / \mathrm{W}$ \\
\hline Fiber effective core area $\left(A_{e f f}\right)$ & $80 \mu \mathrm{m}^{2}$ \\
\hline Fiber nonlinearity coefficient at $\lambda_{3}\left(\gamma=2 \pi n_{2} / \lambda / A_{e f f}\right)$ & $1.32 \mathrm{~W}{ }^{-1} \mathrm{~km}$ \\
\hline Fiber power attenuation coefficient $(\alpha)$ & $0.21 \mathrm{~dB} / \mathrm{km}^{-1}$ \\
\hline Fiber length & $80 \mathrm{~km}$ \\
\hline Receiver equivalent thermal noise & $10 \mathrm{pA} / \sqrt{ } \mathrm{Hz}$ \\
\hline
\end{tabular}

The total power fluctuation induced by XPM in a probe channel at the output of a fiber link can be written as $\widetilde{P}_{\mathrm{XPM}, \text { total }}(\Omega)=\sum_{k \neq b} \widetilde{P}_{\mathrm{XPM}, b k}(\Omega)$ with each individual $\widetilde{P}_{\mathrm{XPM}, b k}(\Omega)$ given by $(20)$ or $(21)$. When a random phase relationship of the modulating signals in each wavelength is assumed, the total XPM-induced XT in a probe channel can be expressed by

$$
X T_{\mathrm{XPM}, \text { total }}=\sum_{k \neq b}\left|\frac{\tilde{P}_{\mathrm{XPM}, b k}(\Omega)}{\tilde{P}_{b}(\Omega) \cdot e^{-\alpha L}}\right|^{2} .
$$

\section{Transmission Link Performance Due to XPM: Simulation, Numerical, and Theoretical Results}

To validate the theoretical results on XPM-induced XT, we carried out numerical calculations (based on the Runge-Kutta method) and computer simulations (based on PTDS) on a system with parameters shown in Table III. The system is a 64-ch 155-Msymbol/s QPSK USB/SCM transmission system over $80 \mathrm{~km}$ of conventional SMF and has a theoretical transport capacity of $20 \mathrm{~Gb} / \mathrm{s}$ per wavelength.

1) Two Wavelengths: Assuming a pump wavelength is modulated by 64-ch QPSK signals occupying a frequency range beyond an octave, the distribution of XT at different RF frequencies in a copropagating probe wavelength is shown in Fig. 8. Three average optical power levels per wavelength were studied: 0,6 and $10 \mathrm{dBm}$. Simulation results are shown by circles $\left(\Delta \lambda_{b p}=-0.2 \mathrm{~nm}\right.$, i.e., $\left.\lambda_{b}-\lambda_{p}=-0.2 \mathrm{~nm}\right)$ or triangles $\left(\Delta \lambda_{b p}=+0.2 \mathrm{~nm}\right.$, but only for input power of 0 $\mathrm{dBm}$ per wavelength). Note that in the simulation, the digital modulation data among different RF channels were carefully kept independent. In addition, we used a time window of

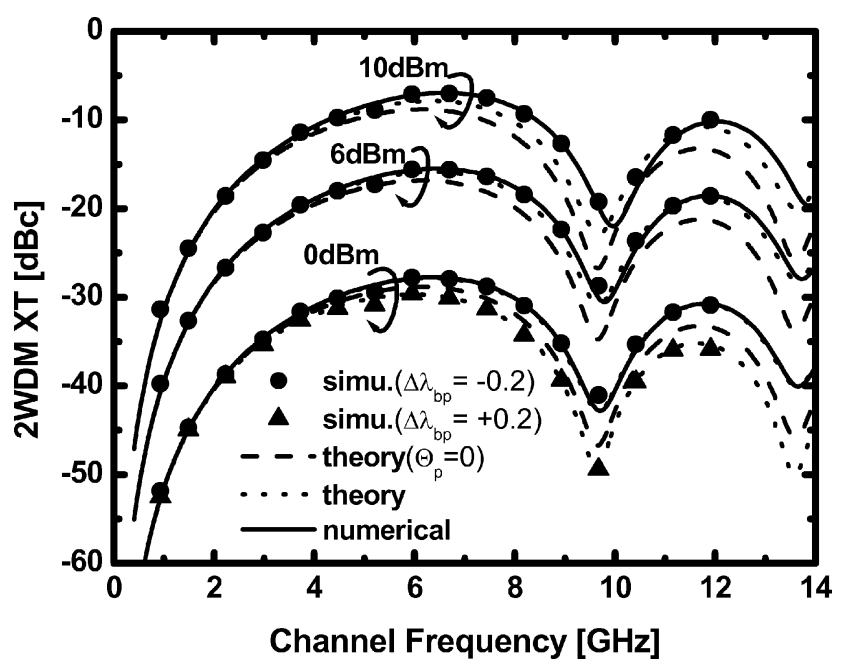

Fig. 8. Two-wavelength XT versus pump modulation frequency for an average optical power per wavelength of 0,6 , or $10 \mathrm{dBm}$. Circular $\left(\Delta \lambda_{b p}=-0.2 \mathrm{~nm}\right)$ and triangular $\left(\Delta \lambda_{b p}=+0.2 \mathrm{~nm}\right)$ symbols are simulation results; dashed $\left(\Theta_{p}=0\right)$ and dotted $\left(\Theta_{p} \neq 0\right)$ lines show the theoretical predictions; solid lines are numerical results. The pump wave is modulated with 64 QPSK channels between 0.93 and $12.648 \mathrm{GHz}$ using USB/SCM.

$128 / 155 \mathrm{e} 6 \mathrm{~s}$, and a sample rate of $8 \times 8 \times 64 \times 155 \mathrm{e} 6 \mathrm{~Hz}$ $(634.88 \mathrm{GHz})$. Numerical results obtained from (20) and the coupled nonlinear Schrödinger equations (16) and (17) are shown by solid lines. Theoretical results obtained from (21) $\left(\Theta_{p} \neq 0\right.$, dotted line; $\Theta_{p}=0$, dashed line) are also shown in Fig. 8.

Several observations from Fig. 8 are hereafter summarized.

- The numerical results show an excellent agreement with those of simulations, even in the case of a high optical power level of $10 \mathrm{dBm}$ per wavelength. In general, the $\mathrm{XT}$ is nearly proportional to $\Omega^{4}$ at low modulation frequencies and nearly proportional to the square of $\bar{P}_{b}(0)$, as predicted by (22).

Theoretical results based on (21) $\left(\Theta_{p} \neq 0\right)$ agree very well with simulation results (within $\sim 2 \mathrm{~dB}$ ). The $\sim 2$-dB discrepancy occurs only at the case of $10 \mathrm{dBm} / \lambda$ with a modulation frequency $>\sim 8 \mathrm{GHz}$. This discrepancy may be attributed to two factors. The first is that at a very high input power per wavelength, the linear-systemapproximated IM-FTF $H_{F p}(z, \Omega)=\cos \left(\Theta_{p} z\right) \pm j$. $\sin \left(\Theta_{p} z\right)$ is no longer valid. This is because the interaction between SPM and chromatic dispersion could generate a second residual optical sideband [7], causing two optical sidebands to interfere with each other at the receiver. The second is that a high input power could cause enhanced IM-IM and PM-IM conversions, and in addition, the fading-induced dip frequency could shift toward slightly higher frequencies [7], [8], [16].

- The theoretical approximation based on (21) $\left(\Theta_{p}=0\right)$ is seen to cause more errors in the higher modulation frequency range when compared with the more exact theoretical estimation based on $\Theta_{p} \neq 0$.

- At a launched power of $0 \mathrm{dBm}$ per wavelength, the XT of $\Delta \lambda_{b p}=-0.2 \mathrm{~nm}$ is always greater than that of $\Delta \lambda_{b p}=+0.2 \mathrm{~nm}$ by up to $\sim 5 \mathrm{~dB}$. The difference is more pronounced at higher modulation frequencies. 


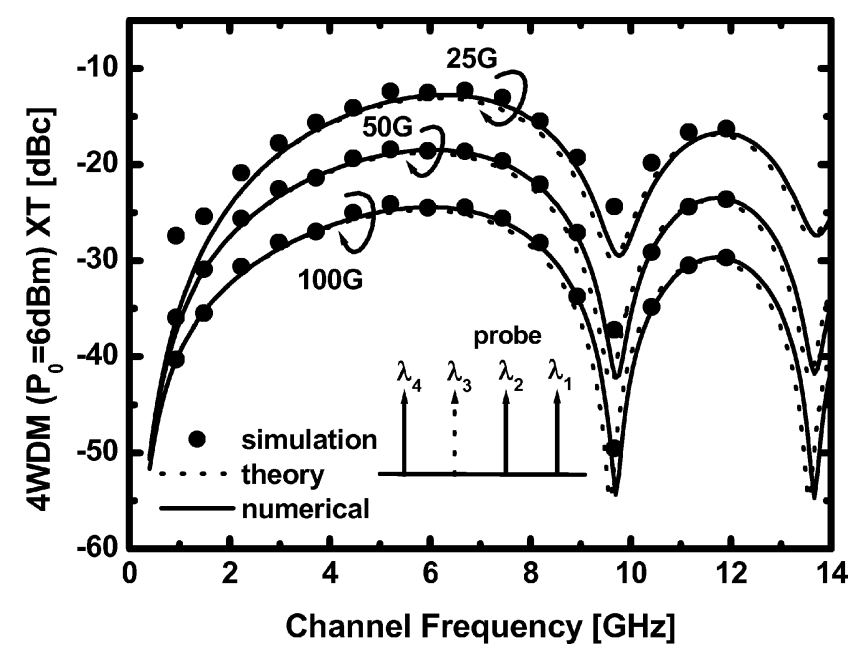

Fig. 9. Four-wavelength XT versus pump modulation frequency for wavelength separations of 25,50 , and $100 \mathrm{GHz}$ (with the probe channel in the center $\left(\lambda_{3}\right)$. Circular, dotted, and solid lines show the simulation, theoretical, and numerical results, respectively. Three pump wavelengths are simultaneously modulated by three uncorrelated 64 QPSK channels (between 0.93 and $12.648 \mathrm{GHz}$ ) using $\mathrm{USB} / \mathrm{SCM}$. The average optical power per wavelength is $6 \mathrm{dBm}$.

This confirms what we said previously about the pump phase change along the fiber (caused by the combined effects of SSB/SCM modulation and GVD) being able to enhance or mitigate the walkoff effect. Since we used USB/SCM here, the walkoff effect will be affected via $\alpha-j\left(\Omega d_{b p}+\Theta_{p}\right)$ (see (21)). Considering a $1550-\mathrm{nm}$ wavelength propagating in a conventional SMF, the dispersion is positive, and therefore $\Theta_{p}$ is positive. Consequently, a negative $\Delta \lambda_{b p}\left(\Omega d_{b p}<0\right)$ will generate more XPM-induced XT than a positive $\Delta \lambda_{b p}\left(\Omega d_{b p}>0\right)$, because the latter enhances the walkoff effect.

- The CNR requirement of a QPSK signal is 17 and $20 \mathrm{~dB}$ in theory and practice, respectively. Therefore, the power level per wavelength should be kept below $\sim 3 \mathrm{dBm}$ in this transmission system.

- A dip frequency occurs at somewhere between 9.6 and $9.8 \mathrm{GHz}$ under different conditions. This is due to the fact that PM-IM conversion vanishes around $9.6 \mathrm{GHz}$ for a SMF fiber length of $80 \mathrm{~km}$, i.e., $\Theta_{b} z=n \pi$ in $C_{\mathrm{PM}-\mathrm{IM}, b}(z, \Omega)=-\sin \left(\Theta_{b} z\right)$, and therefore the XT performance is improved.

2) Four Wavelengths: Fig. 9 shows the XPM-induced XT due to four adjacent wavelengths (spaced at 25,50 , or $100 \mathrm{GHz}$ ) for the system given in Table III. The average optical power per wavelength is $6 \mathrm{dBm}$. The probe channel is located at the center $\left(\lambda_{3}\right.$ in the inset of Fig. 9). Simulation results are shown in circles, numerical results (using (16), (17), (20), and (24)) are shown in solid lines, and theoretical results (using (21) and (24)) are shown in dotted lines. Several observation can be made regarding Fig. 9.

- All results from simulations, numerical calculations, and theory match very well. However, it should be noted that at higher power levels (e.g., $10 \mathrm{dBm}$ ), the influence of fiber nonlinearity on the PM-IM conversion becomes nonneg- ligible, and the small-signal analysis results used to derive (21) may no longer be valid.

- The XT is nearly inversely proportional to the square of wavelength separation for moderate to high frequencies, as predicted by (22).

- It can be predicted that the XT estimates based on either $\Theta_{p} \neq 0$ or $\Theta_{p}=0$ (not shown in figure) will have a negligible difference (within $1 \mathrm{~dB}$ for 0.2 -nm wavelength separation). This is because, from the viewpoint of a central probe channel, the mitigated or enhanced XTs due to leftor right-hand-side neighbor channels are averaged out.

\section{Complete System Performance Considering AlL TRANSMISSION IMPAIRMENTS}

In this section, we shall include all possible transmission impairments, in addition to the XPM-induced XT described in the last section. These impairments include receiver thermal noise, signal-spontaneous and spontaneous-spontaneous beat noise due to optical amplifiers, shot noise, and linear-fiber-dispersion-induced nonlinear distortions (CSOs and CTBs, which were discussed in Section IV). Note that in the 64-ch QPSK system example shown in Table III, only CSOs need to be considered in the transmission system when OMI per channel is $\ll 1$ (this is because CSO $\sim m^{2}$ and CTB $\sim m^{4}$, thus CTB $\ll$ CSO). However, if we consider a system with modulation frequencies distributed within an octave, e.g., 32-ch $155 \mathrm{Msymbol} / \mathrm{s}$ 16-QAM (or QPSK) between 6.882 and 12.648 $\mathrm{GHz}$, then only the CTB needs to be considered (because all CSOs are outside the frequency range under consideration). As we will see, keeping all modulation channels within an octave (and avoiding CSO) is a way to achieve high-capacity, long-distance transmission without dispersion compensation.

We used an erbium-doped fiber amplifier (EDFA) as a preamplifier in the system so that the receiver thermal noise is not dominant [21]. The preamplifier has an unsaturated gain of 30 $\mathrm{dB}$, a 3-dB saturation output power of $10 \mathrm{dBm}$, and a noise figure of $5 \mathrm{~dB}$.

\section{A. 64 Channels of QPSK}

Four wavelengths, each carrying the same 64-ch QPSK signals (see Table III) are considered in an 80-km transmission system. No dispersion compensation is used. The probe wavelength again is $\lambda_{3}$.

Fig. 10 shows the theoretical results of the worst-case NSR (the worst among 64 subcarrier channels) as a function of rms OMI per channel. Note that in the case of four wavelengths, the worst-case channel frequency depends on the combination of CSO and XPM-induced XT. The dashed line represents the NSR caused by linear -fiber-dispersion-induced CSOs, which was obtained from (12). It shows that the NSR is proportional to the square of rms OMI per channel. Due to this linear-dispersion-limited NSR, we must have a low OMI per channel in order to obtain a low NSR, e.g., an OMI per channel of less than $1.5 \%$ to obtain NSR $<-20 \mathrm{~dB}$. However, to reach a long transmission distance with such a small OMI per channel, the launched optical power per wavelength has to be high. When there is only 


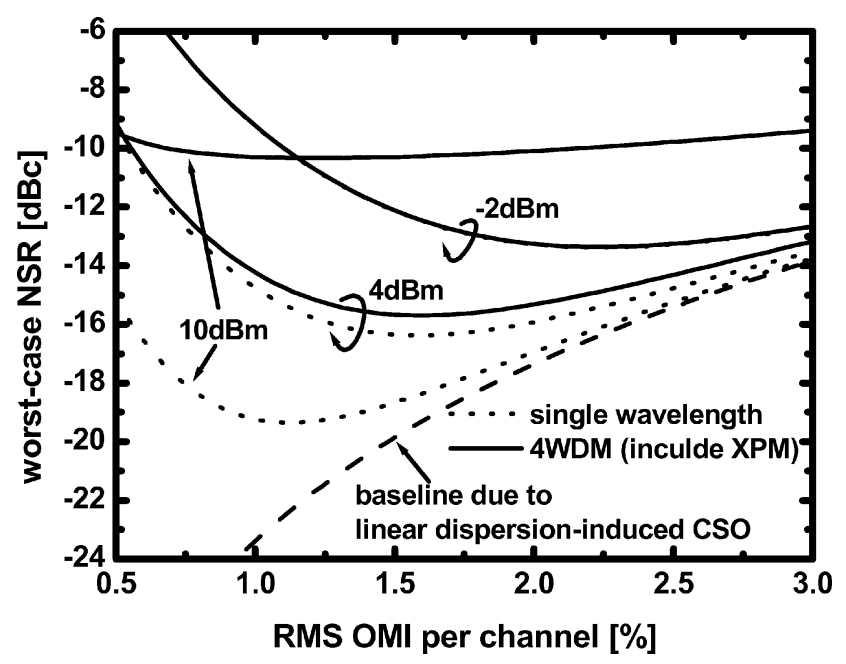

Fig. 10. Theoretical results of the worst-channel NSR versus the rms OMI per channel for an average optical power per wavelength of $-2,4$, and $10 \mathrm{dBm}$, for the system given in Table III. Dotted lines are for single wavelength, while solid lines are for four wavelengths. The wavelength separation is $50 \mathrm{GHz}$. The dashed line is due to linear-fiber-dispersion-induced CSO.

a single wavelength, we see from the three dotted lines in Fig. 10 that the launched optical power has to be as high as $10 \mathrm{dBm}$ so that NSR can reach its best value of $-20 \mathrm{~dB}$ to meet the requirement of a QPSK signal. We also notice that there is an optimum OMI per channel, i.e., $2.2 \%, 1.6 \%$, and $1.1 \%$, for the three power levels, respectively. When the OMI per channel is low, e.g., lower than $1 \%$, the dominant noise is the signal-spontaneous beat noise, which is linearly proportional to the signal power. When the OMI per channel is high, e.g., higher than $2 \%$, the dominant noise is the linear-dispersion-induced CSO.

In the case of four wavelengths, XPM-induced XT has to be included, and the theoretical results based on (21) and (24) are shown by solid lines in Fig. 10. Note that the wavelength separation is $50 \mathrm{GHz}$, and the probe wavelength is at the center of the four wavelengths, i.e., $\lambda_{3}$. As shown in (20), XPM-induced XT is proportional to the square of optical power per wavelength; thus, a 10-dBm input power per wavelength causes a significant degradation to the single-wavelength NSR. When the input optical power is $-2 \mathrm{dBm}$ per wavelength, the theoretical results are the same for both single and four wavelengths. This is because the XPM-induced XT is negligible when compared with the combination of fiber-dispersion-induced CSOs and signal-spontaneous beat noise. When the input power is 4 $\mathrm{dBm}$ per wavelength, the NSR is only slightly degraded by the XPM-induced XTs.

Fig. 11 shows the distribution of NSR in all the RF channels $(\mathrm{OMI} / \mathrm{ch}=1.8 \%)$ given in Table III, when there are four equal-power wavelengths with per wavelength power of -2 , 4 , or $10 \mathrm{dBm}$ and a channel spacing of $50 \mathrm{GHz}$. The theoretical results (dotted lines) are based on (12), (21), (24), and various receiver noise terms. Again, the NSR limited by the linear-fiber-dispersion-induced CSOs (see (12)) serves as the fundamental limitation of the given transmission system despite of the wavelength power level. We see from Fig. 11 that it is impossible for all QPSK channels to reach the minimum practical requirement of $-20 \mathrm{~dB}$ NSR after $80 \mathrm{~km}$ of transmis-

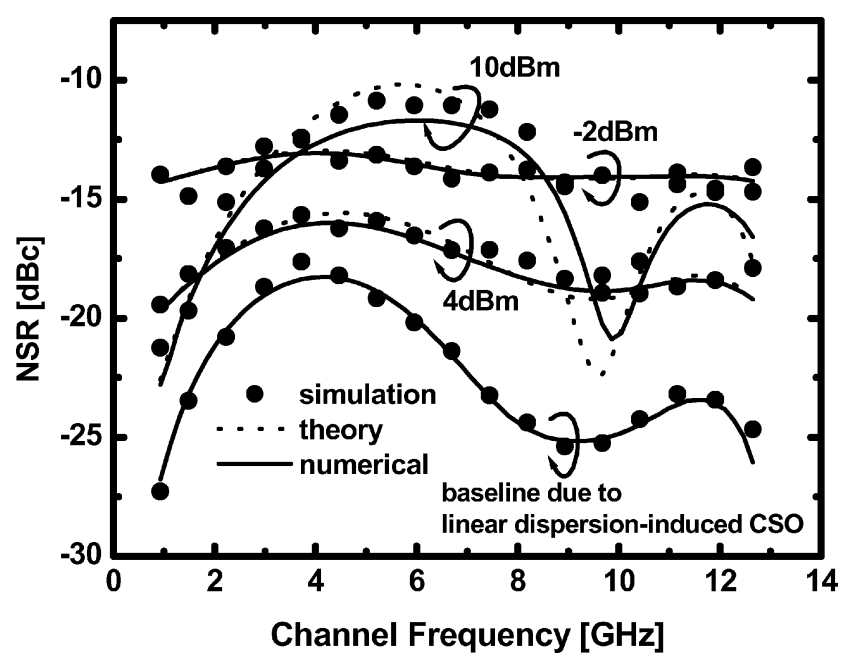

Fig. 11. NSR versus channel frequency in the case of four wavelengths for the system given in Table III. Average optical power per wavelength is $-2,4$, or $10 \mathrm{dBm}$. Circular symbols are simulation results, dotted lines are theoretical results, and solid lines are numerical results. The wavelength separation is 50 $\mathrm{GHz}$, and the rms OMI per channel is $1.8 \%$.

sion without dispersion compensation unless FEC coding gain is available (see Table IV).

Also shown in the figure are the simulation (circle) and numerical (solid line) results, which exhibit excellent agreement over the entire frequency range of the subcarrier channels for all optical powers. Theoretical results at $10 \mathrm{dBm}$, however, show a slight discrepancy $(\sim 2 \mathrm{~dB})$ from the simulation results. This is because the high-power-induced fiber nonlinearity affects the applicability of the linear-system IM-FTF, as was discussed in Section VI-A. When the input power per wavelength is $4 \mathrm{dBm}$, we can see in Fig. 11 that the worst-channel NSR is about $-16 \mathrm{~dB}$ and is consistent with what was presented in Fig. 10.

Table IV summarizes the theoretical results based on the optimum-input optical power per wavelength, rms OMI per channel, and the corresponding worst-case NSR for a wavelength spacing of 25,50 , and $100 \mathrm{GHz}$, respectively. It can be seen that the wider the wavelength spacing, the higher input optical power per wavelength can be launched into the system, the lower OMI per channel can be used, and a better NSR performance can be obtained.

The coding gains required to reach a BER of $10^{-12}$ in practice for all wavelength spacings are also shown. These coding gains should be within the reach of conventional Reed-Solomon FEC codecs. For longer transmission distance, more optical amplifier-induced noise will have to be added, and the coding gain may not be enough to cope with this additional noise due to longer transmission. Setting the total modulation bandwidth to within an octave may be a good solution to this problem. The example in the following subsection confirms the feasibility of this approach.

\section{B. 32 Channels of 16-QAM or QPSK}

Four wavelengths, each carrying 32-ch $155 \mathrm{Msymbol} / \mathrm{s}$ 16-QAM (or QPSK) signals equally distributed between 6.882 and $12.648 \mathrm{GHz}$, are considered in an $80-\mathrm{km}$ transmission 
TABLE IV

For the System Given in Table III, a Summary of the Optimum OPTICAl Power, rms OMI PeR ChanNel and the CORRESPONDING WORST-CASE NSR IN a TRANSMisSion System With Four MultipleXed WaVelengths. Three-Wavelength Spacings ARe Given: 25, 50, AND $100 \mathrm{GHz}$. The Required Coding Gains to Reach a BER of $10^{-12}$ Are Also Shown

\begin{tabular}{c|c|c|c}
\hline & $25 \mathrm{GHz}(0.2 \mathrm{~nm})$ & $50 \mathrm{GHz}(0.4 \mathrm{~nm})$ & $100 \mathrm{GHz}(0.8 \mathrm{~nm})$ \\
\hline $\begin{array}{c}\text { Optimum optical power } \\
\text { per wavelength }(\mathrm{dBm})\end{array}$ & 2.24 & 4.48 & 6.81 \\
\hline $\begin{array}{c}\text { Optimum RMS OMI } \\
\text { per channel }(\%)\end{array}$ & 1.78 & 1.55 & 1.36 \\
\hline Worst-channel NSR (dB) & -14.66 & -15.72 & -16.88 \\
\hline $\begin{array}{c}\text { Coding gain required to } \\
\text { reach BER }=10^{-12}(\mathrm{~dB}) \text { in practice* }\end{array}$ & 5.34 & 4.28 & 3.12 \\
\hline For QPSK, NSR $<\sim-16.95 \mathrm{~dB}$ in theory and $-20 \mathrm{~dB}$ in practice is required to obtain a BER $=10^{-12}$
\end{tabular}

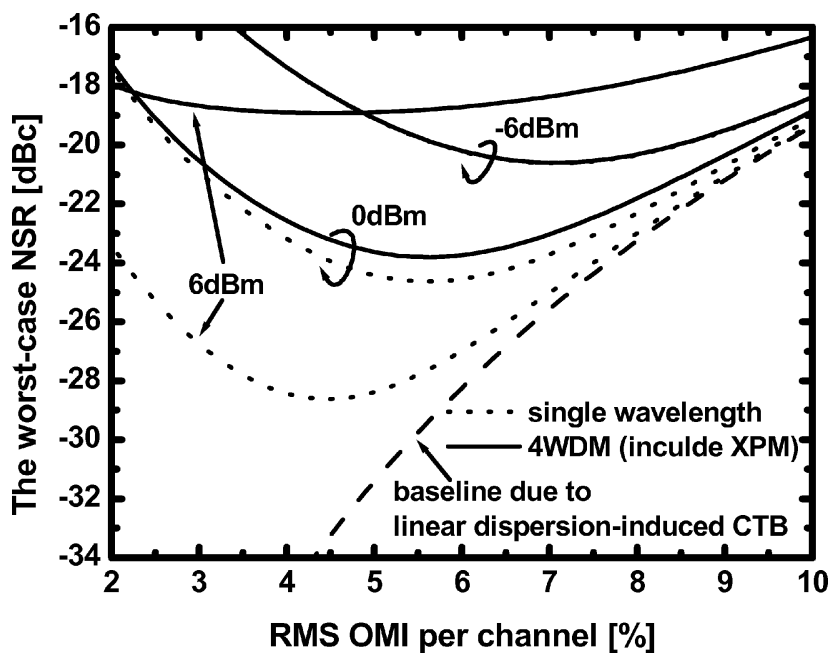

Fig. 12. Theoretical results of the worst-channel NSR versus the rms OMI per channel for an average optical power per wavelength of $-6,0$, and $6 \mathrm{dBm}$. Dotted lines are for the case of single wavelength, while solid lines are for the case of four wavelengths. The wavelength separation is $50 \mathrm{GHz}$. The dashed line is due to linear-fiber-dispersion-induced CTB.

system. No dispersion compensation is used. The probe wavelength again is $\lambda_{3}$.

Fig. 12 shows the theoretical results of the worst-case NSR as a function of rms OMI per channel. The dashed line represents the NSR caused by linear-fiber-dispersion-induced third-order nonlinear distortions, which were obtained from (15). It shows that the NSR is proportional to $m^{4}$. It is obvious that we can now work in a region with much higher OMI per channel than that in Fig. 10. To reach an NSR $<-27 \mathrm{~dB}$ for practical 16-QAM signals without coding gain, the OMI per channel needs to be less than $6.5 \%$. When there is only a single wavelength, we see from the three dotted lines in Fig. 12 (for a launched power of $-6,0$, and $6 \mathrm{dBm}$ per wavelength) that the launched optical power has to be as high as $6 \mathrm{dBm}$ such that NSR can reach its best value of $-29 \mathrm{~dB}$ to meet the requirement of a 16-QAM signal. We also notice that there is an optimum OMI per channel, i.e., $7.1 \%, 5.6 \%$, and $4.5 \%$, for the three power levels, respectively. When the OMI per channel is low, e.g., lower than $4 \%$, the dominant noise is the signal-spontaneous beat noise, which is linearly proportional to the signal power. When the OMI per

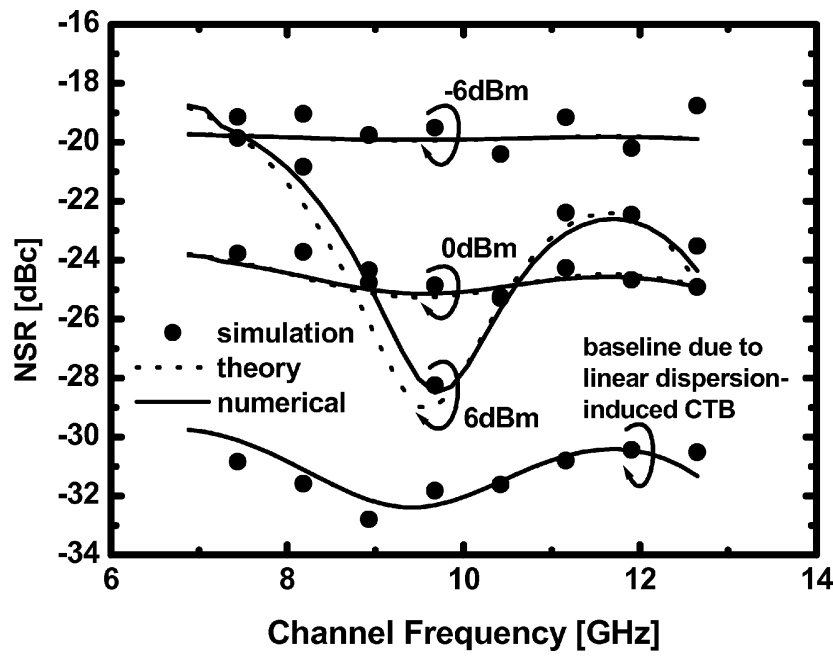

Fig. 13. NSR versus the channel frequency in the case of four wavelengths. Average optical power per wavelength is $-6,0$, or $6 \mathrm{dBm}$. Circular symbols are simulation results, dotted lines are theoretical results, and solid lines are numerical results. The wavelength separation is $50 \mathrm{GHz}$, and the rms OMI per channel is $5.5 \%$.

channel is high, e.g., higher than $7 \%$, the dominant noise is the linear-dispersion-induced CTB.

In the case of four wavelengths, XPM-induced XT is included, and the theoretical results based on (21) and (24) are shown by solid lines in Fig. 12. We can make several observations.

1) When the input optical power is as low as $-6 \mathrm{dBm}$ per wavelength, the theoretical results are the same for both cases of single and four wavelengths, because the XPMinduced XT is negligible.

2) When the input optical power is as high as $6 \mathrm{dBm}$ per wavelength, the XPM-induced XT degrades the singlewavelength NSR severely.

3) When the input power is $0 \mathrm{dBm}$ per wavelength, the NSR is only slightly degraded by the XPM-induced XTs.

Fig. 13 shows the distribution of NSR in all the RF channels $(\mathrm{OMI} / \mathrm{ch}=5.5 \%)$, when there are four equal-power wavelengths with per wavelength power of $-6,0$, or $6 \mathrm{dBm}$ and a channel spacing of $50 \mathrm{GHz}$. The theoretical results (dotted lines) are based on (15), (21), (24), and additive receiver noise terms. 
TABLE V

For 32 Channels of 16-QAM Signals EOUally Distributed Between 6.882 and $12.648 \mathrm{GHz}$ (Same Transport Capacity as That Given in Table III), a Summary of the Optimum OPTICAL POWER, rms OMI per CHANNEL, AND THE CORRESPONDING WORST-CASE NSR IN a Transmission System With Four Multiplexed WaVelengths. Three-WaVelength Spacings Are Studied: 25, 50, and $100 \mathrm{GHz}$. The ReQuired Coding Gains to REACH A BER OF $10^{-12}$ FOR QPSK AND 16-QAM ARE ALSO SHOWN

\begin{tabular}{l|c|c|c}
\hline & $25 \mathrm{GHz}(0.2 \mathrm{~nm})$ & $50 \mathrm{GHz}(0.4 \mathrm{~nm})$ & $100 \mathrm{GHz}(0.8 \mathrm{~nm})$ \\
\hline $\begin{array}{c}\text { Optimum optical power } \\
\text { per wavelength }(\mathrm{dBm})\end{array}$ & -1.49 & 0.75 & 3.01 \\
\hline $\begin{array}{c}\text { Optimum RMS OMI } \\
\text { per channel }(\%)\end{array}$ & 5.94 & 5.45 & 5 \\
\hline Worst-channel NSR (dB) & -22.39 & -23.88 & -25.39 \\
\hline $\begin{array}{c}\text { Coding gain required to } \\
\text { reach BER }=10^{-12}(\mathrm{~dB}) * \text { for QPSK }\end{array}$ & 0 & 0 & 0 \\
\hline $\begin{array}{c}\text { Coding gain required to } \\
\text { reach BER }=10^{-12}(\mathrm{~dB}) * * \text { for } 16-\mathrm{QAM}\end{array}$ & 4.61 & 3.12 & 1.61 \\
* For QPSK, NSR $<\sim-16.95 \mathrm{~dB}$ in theory and $-20 \mathrm{~dB}$ in practice is required to obtain a BER $=10^{-12}$ \\
** For 16-QAM, NSR $<\sim-23.88 \mathrm{~dB}$ in theory and $-27 \mathrm{~dB}$ in practice is required to obtain BER $=10^{-12}$
\end{tabular}

The NSR limited by the linear-fiber-dispersion-induced CTBs (see (15)) serves as the fundamental limitation of the transmission system, which gives an NSR limit of about $-30 \mathrm{~dB}$ after $80-\mathrm{km}$ transmission. If we carefully choose $0 \mathrm{dBm}$ as the per-wavelength power level to avoid XPM-induced XT, the resultant NSR is about $-24 \mathrm{~dB}$ and is limited by the amplifier signal-spontaneous beat noise. This NSR is more than sufficient for QPSK signals but requires $>3 \mathrm{~dB}$ FEC gain for 16-QAM signals.

Also shown in the figure are the simulation (circle) and numerical (solid line) results, which exhibit excellent agreement over the entire frequency range of the subcarrier channels for all optical powers. Theoretical results at $6 \mathrm{dBm}$, however, show a slight discrepancy $(\sim 1 \mathrm{~dB})$ from the simulation results because of the accuracy of the theoretical prediction of XPM-induced XT due to high-power-induced fiber nonlinearity. When the input power per wavelength is $0 \mathrm{dBm}$, we can see in Fig. 13 that the worst-channel NSR is about $-24 \mathrm{~dB}$ and is consistent with what was presented in Fig. 12.

Table V summarizes the theoretical results based on the optimum-input optical power per wavelength, the rms OMI per channel, and the corresponding worst-case NSR for a wavelength spacing of 25,50 , and $100 \mathrm{GHz}$, respectively. It can be seen that the wider the wavelength spacing, the higher the input optical power per wavelength can be launched into the system, the lower the rms OMI per channel can be used, and a better NSR performance can be obtained. Also note that the worst-channel NSR can get 7.7-8.5-dB improvement than those given in Table IV. This implies that we can comfortably increase the constellation to 16-QAM, while maintaining the same system capacity $(20 \mathrm{~Gb} / \mathrm{s})$ and transmission distance. On the other hand, if we decrease the transmission capacity to $10 \mathrm{~Gb} / \mathrm{s}$ by using 32 channels of $155 \mathrm{Ms} / \mathrm{s}$ QPSK within an octave, we can obtain a much longer transmission distance than $80 \mathrm{~km}$ because now the main system limitation comes from amplifier signal-spontaneous beat noise, just as in conventional transmission systems.
VIII. CONCLUSION

This paper investigated the fundamental linear and nonlinear system limitations of multichannel SSB/SCM/DWDM systems through theoretical analysis, numerical calculations, and computer simulations. The paper also presented general analytical tools that can be used in either wireless or metro optical systems that transport multiple SSB/SCM narrow-band digital signals on a single wavelength or multiple wavelengths. These analytical tools include the following: (11) and Table I for MZI-modulator-induced CTB; (12) and Table II for CSOs caused by linear fiber dispersion; (13)-(15) for CTBs caused by linear fiber dispersion; and (21)-(24) for XPM-induced XT. To predict XPMinduced XT precisely and without any limitation by wavelength spacing or modulation frequency range, (20) was derived for numerical calculations.

Through case studies of 64 or 32 channels of 155 Msymbol/s $n$-QAM signals, several system considerations for SSB/SCM/DWDM transmissions were discovered.

1) The MZI modulator nonlinear transfer curve-induced CTB is the very basic SSB/SCM system limitation even when all fiber dispersion has been completely compensated. This CTB is usually not of concern because it is relatively low, e.g., lower than $-30 \mathrm{dBc}$ (64 channels) and $-50 \mathrm{dBc}$ (32 channels) in the case studies.

2) Although an SSB/SCM system does not have the same distance-dependent carrier-suppression problem as in a conventional double-sided SCM system, its transmission distance is still severely limited by linear-fiber-dispersion-induced CSOs when the multiple modulation signals occupy more than an octave of frequency range. In the case study of 64 channels of QPSK signals with 4\% OMI per channel, this CSO can be as high as $-17 \mathrm{dBc}$ and $-9 \mathrm{dBc}$ when the transmission distances are $\sim 50$ and $>150 \mathrm{~km}$, respectively. Beyond $150 \mathrm{~km}$, this CSO saturates at $-9 \mathrm{dBc}$.

3) The main optical fiber nonlinearity-induced impairment in a DWDM SSB/SCM system is caused by XPM. To 
avoid this XPM-induced XT, the launched optical power per wavelength should be kept below a certain level (about $3 \mathrm{dBm}$ in the case studies).

4) Two case studies of $20 \mathrm{~Gb} / \mathrm{s}$ per wavelength over an 80-km DWDM system without dispersion compensation were carried out. It was found that both are theoretically achievable but with little margin left, especially when wavelength channel spacing is small. However, it was noticed that it is possible to transport $10 \mathrm{~Gb} / \mathrm{s}$ (e.g., 32 $155 \mathrm{Msymbol} / \mathrm{s}$ QPSK signals with traditional FEC and within an octave) per wavelength using SSB/SCM in a DWDM system, even at $25-\mathrm{GHz}$ wavelength spacing, over a transmission distance of many hundreds of kilometers without dispersion compensation. The key point in achieving this superior transmission performance is to keep all the modulation frequencies within an octave so that the dominant transmission impairment is the linear-dispersion-induced CTB.

\section{REFERENCES}

[1] G. H. Smith, D. Novak, and Z. Ahmed, "Technique for optical SSB generation to overcome dispersion penalties in fiber-radio systems," Electron. Lett., vol. 33, pp. 74-75, Jan. 1997.

[2] G. H. Smith and D. Novak, "Broad-band millimeter-wave (38 GHz) fiber-wireless transmission system using electrical and optical SSB modulation to overcome dispersion effects," IEEE Photon. Technol. Lett., vol. 10, pp. 141-143, Jan. 1998.

[3] F. Ramos and J. Marti, "Comparison of optical single-sideband modulation and chirped fiber gratings as dispersion mitigating techniques in optical millimeter-wave multichannel systems," IEEE Photon. Technol. Lett., vol. 11, pp. 1479-1481, Nov. 1999.

[4] M. R. Phillips and D. M. Ott, "Crosstalk due to optical fiber nonlinearities in WDM CATV lightwave systems," J. Lightwave Technol., vol. 17, pp. 1782-1792, Oct. 1999.

[5] M.-C. Wu, P.-Y. Chiang, and W. I. Way, "On the validity of using CW tones to test the linearity of multichannel M-QAM subcarrier multiplexed lightwave systems," IEEE Photon. Technol. Lett., vol. 12, pp. 413-415, Apr. 2000.

[6] H. Schmuck, "Comparison of optical millimeter-wave system concepts with regard to chromatic dispersion," Electron. Lett., vol. 31, no. 21, pp. 1848-1849, Oct. 1995.

[7] F. Ramos and J. Marti, "Frequency transfer function of dispersive and nonlinear single-mode optical fibers in microwave optical systems," IEEE Photon. Technol. Lett., vol. 12, pp. 549-551, May 2000.

[8] P. Laurêncio and M. C. R. Medeiros, "Dynamic range of optical links employing optical single side-band modulation," IEEE Photon. Technol. Lett., vol. 15, pp. 748-750, May 2003.

[9] M. R. Phillips and D. M. Ott, "Crosstalk caused by nonideal output filters in WDM lightwave systems," IEEE Photon. Technol. Lett., vol. 12, pp. 1094-1096, Aug. 2000.

[10] A. Cartaxo, "Impact of modulation frequency on cross-phase modulation effect in intensity modulation-direct detection WDM systems," IEEE Photon. Technol. Lett., vol. 10, pp. 1268-1270, Sept. 1998.

[11] — " "Cross-phase modulation in intensity modulation-direct detection WDM systems with multiple optical amplifiers and dispersion compensators," J. Lightwave Technol., vol. 17, pp. 178-190, Feb. 1999.

[12] J. Wang and K. Petermann, "Small signal analysis for dispersive optical fiber communication systems," J. Lightwave Technol., vol. 10, pp. 96-100, Jan. 1992.
[13] A. Røyset, L. Bjerkan, D. Myhre, and L. Hafskjær, "Use of dispersive optical fiber in the characterization of chirp in semiconductor lasers," Electron. Lett., vol. 30, no. 9, pp. 710-712, Apr. 1994.

[14] L. Bjerkan, A. Røyset, L. Hafskjær, and D. Myhre, "Measurement of laser parameters for simulation of high-speed fiberoptic systems," $J$. Lightwave Technol., vol. 14, pp. 839-850, May 1996.

[15] A. Røyset, L. Bjerkan, and A. Sudb, "Compensation of optical fiber dispersion in the electrical domain for transmission systems with direct detection," Electron. Lett., vol. 30, no. 2, pp. 152-153, Jan. 1994.

[16] A. Cartaxo, B. Wedding, and W. Idler, "Influence of fiber nonlinearity on the fiber transfer function: Theoretical and experimental analysis," $J$. Lightwave Technol., vol. 17, pp. 1806-1813, Oct. 1999.

[17] F. Devaux, Y. Sorel, and J. Kerdiles, "Simple measurement of fiber dispersion and of chirp parameter of intensity modulated light emitter," $J$. Lightwave Technol., vol. 11, pp. 1937-1940, Dec. 1993.

[18] A. Cartaxo, B. Wedding, and W. Idler, "Influence of fiber nonlinearity on the phase noise to intensity noise conversion in fiber transmission: Theoretical and experimental analysis," J. Lightwave Technol., vol. 16, pp. 1187-1194, July 1998.

[19] G. P. Agrawal, Nonlinear Fiber Optics, 2nd ed. San Diego, CA: Academic, 1995.

[20] T. K. Chiang, N. Kagi, M. E. Marhic, and L. G. Kazovsky, "Cross-phase modulation in fiber links with multiple optical amplifiers and dispersion compensators," J. Lightwave Technol., vol. 14, pp. 249-260, Mar. 1996.

[21] W. I. Way, Broadband Hybrid Fiber/Coax Access System Technologies. New York: Academic, 1998.

W. H. Chen was born in Chia-Yi, Taiwan, R.O.C., in 1973. He received the B.S. and M.S. degrees from the National Chiao-Tung University, Taiwan, R.O.C., in 1996 and 1998, respectively. He is currently working toward the Ph.D. degree at the same institution.

His research interests focus on transmission limitations on subcarrier-multiplexing/dense-wavelength-division-multiplexing optical communication systems due to fiber nonlinearities.

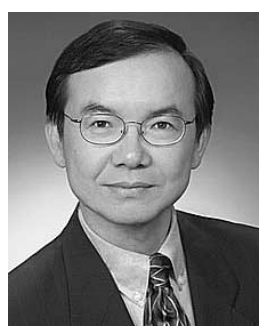

Winston I. Way (S'82-M' 82-SM'88-F'01) received the M.S. and Ph.D. degrees from the University of Pennsylvania, Philadelphia, in 1981 and 1983 , respectively.

He was with Applied Research, Bellcore (now Telcordia), Red Bank, NJ, from 1984 to 1992, where he pioneered research in subcarrier-multiplexed lightwave systems and conducted a number of well-known dense-wavelength-division-multiplexing (DWDM) digital/analog video transmission experiments. From 1992 to 2000, he was a Professor at the National Chiao-Tung University, Hsinchu, Taiwan, R.O.C., where he continued leading many research projects in HFC systems and networks. From 1998 to 2000, he was also a Consultant at Telcordia, conducting research on next-generation Internet optical networks. In 2000, he founded OpVista Inc., Irvine, CA, and has been developing ultra-DWDM transmission equipment. $\mathrm{He}$ is the author of the book Broadband Hybrid/Fiber Coax System Technologies (New York: Academic, 1998), has published more than 100 journal and conference papers, and holds more than a dozen published or pending U.S. patents.

Dr. Way has served on the technical program committees of OFC, MTT, OECC, the IEEE Lasers \& Electro-Optics Society (LEOS), and the Optical Society of America (OSA). 